\title{
TO WHAT EXTENT GAN BLOCKCHAIN HELP DEVELOPMENT CO-OPERATION ACTORS MEET THE 2030 AGENDA?
}

Priscilla Boiardi and Esme Stout
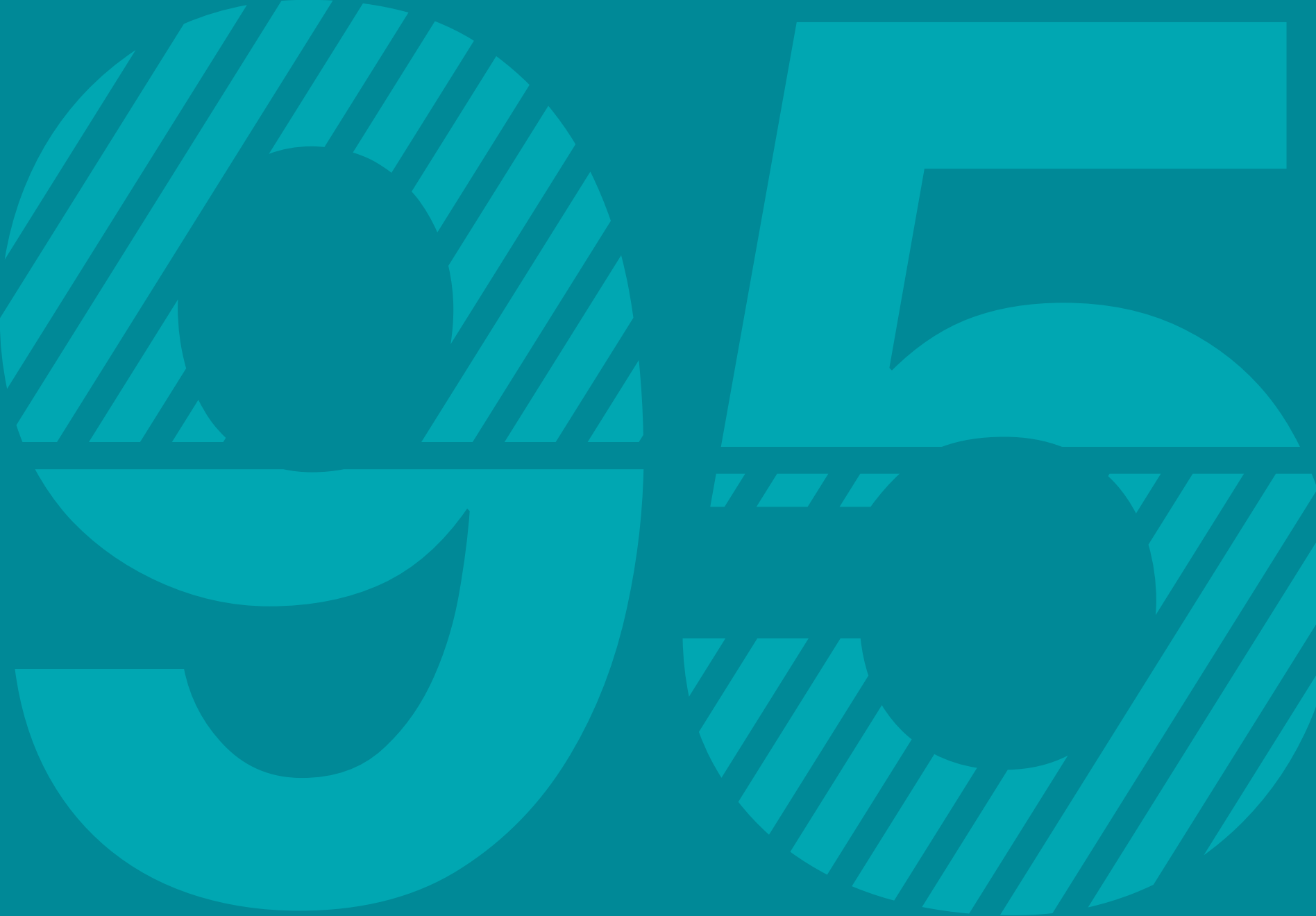

\section{OECD DEVELOPMENT CO-OPERATION WORKING PAPER 95}

Authorised for publication by Jorge Moreira da Silva, Director, Development Co-operation Directorate

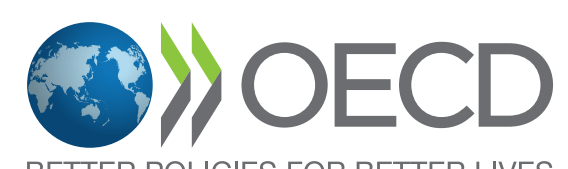



OECD Working Papers do not represent the official views of the OECD or of its member countries. The opinions expressed and arguments employed are those of the authors. Working Papers describe preliminary results or research in progress by the authors and are published to stimulate discussion on a broad range of issues on which the OECD works. Comments on the present Working Paper are welcomed and may be sent to dac.contact@oecd.org — the Development Co-operation Directorate, OECD, 2 rue André Pascal, 75775 Paris Cedex 16, France. This document, as well as any data and any map included herein, are without prejudice to the status of or sovereignty over any territory, to the delimitation of international frontiers and boundaries and to the name of any territory, city or area.

Please cite this paper as Boiardi, P. and Stout, E. (2021) "To what extent can blockchain help development co-operation actors meet the 2030 Agenda?" OECD Development Co-operation Working Papers, No 95, OECD Publishing, Paris. 


\section{Foreword}

Distributed ledger technology (DLT) digitally secures a record of transactions and distributes the subsequent information across a peer-to-peer network. Notably, it does not require the involvement of a third party (such as a bank or government) to operate (Marr, 2020[1]). As a term, DLT covers a broad range of digital database technology implementation, including Holochain and Radix (Khan, 2019[2]). However, for the purpose of this paper, we will be examining DLT's most famous example, blockchain, which came to international prominence following the pseudonymous Satoshi Nakamoto's landmark 2008 whitepaper. This paper differentiates between cryptocurrencies like Bitcoin, which is a type of application built on blockchain, and the underlying technology itself (Mulligan, Godsiff and Brunelle, 2020[3]). While often mistakenly referred to as interchangeable, they are two distinct entities and should be treated as such.

In the years since the Addis Ababa Action Agenda (AAAA) (United Nations, 2015 $5_{[4]}$ ) first called for a new financing framework for sustainable development, a number of agencies, particularly multilaterals, have been experimenting with blockchain technology. For instance, the World Bank established a Blockchain Lab in 2017, and humanitarian organisations such as the United Nations Children's Fund (UNICEF) and the World Food Programme (WFP) continue to explore the benefits of employing blockchain to facilitate the disbursement of cash-based aid and digital identities (Pisa, 2018 $\left.{ }_{[5]}\right]$.

The interest amongst the development community for blockchain stems from its perceived ability to 'solve' problems in contexts characterised by lack of trust, information and power asymmetries, as well as inefficient systems more generally. This interest has also prompted a proliferation of development-related literature on the subject. Amongst others, USAID (Nelson, 2018[6]) and the German Development Agency, Deutsche Gesellschaft für Internationale Zusammenarbeit (GIZ) GmbH (GIZ Blockchain Lab, 2019 $9_{[7]}$ ) have both published primers on blockchain. Elsewhere, the Danish and United Kingdom development agencies published their own documents exploring the ways in which blockchain could be used to enhance aid and development programmes (Coinfy, 2017 ${ }_{[8]}$ ) (Mulligan, 2016[9]). In parallel, the key messages from the annual Blockchain Africa Conference demonstrate that a growing number of developing countries are seeking to be included in this conversation (Move Africa Forward, 2020[10]).

However, the number of blockchain for development use-cases with proven success beyond the pilot stage remain relatively few. The resulting focus on a select number of well-known use cases ultimately limits the practical utility of the blockchain for development mappings that have been undertaken. With this in mind, although this paper surveys the broad landscape of blockchain for development to inform policy makers with limited prior knowledge, it also narrows the lens to critically examine four successful applications (including Seso Global, which has thus far not been widely discussed). The paper outlines key blockchain concepts, alongside the implications of blockchain solutions, to help policymakers be more realistic and discriminating, as well as optimise development impact and minimise harm. As part of the on-going work by the OECD's Blockchain Policy Centre, this paper asserts that post-COVID-19, Development Assistance Committee (DAC) donors and their development partners have a unique opportunity to shape the use of blockchain. This is particularly timely as the global adoption of emerging technologies is set to accelerate post-COVID-19. 


\section{Acknowledgements}

This paper was prepared by the OECD Development Co-operation Directorate (DCD) under Director Jorge Moreira da Silva.

Priscilla Boiardi, Policy Analyst, and Esme Stout, Junior Policy Analyst, were the lead authors of this publication, under the supervision of Paul Horrocks, Head of the Private Finance for Sustainable Development Unit, and under the strategic leadership of Haje Schütte, Head of Financing for Sustainable Development Division.

The authors would like to extend special thanks to Sai Aashirvad Konda, who contributed significantly to the research and drafting of this working paper, including the detailed expert interviews.

The authors are also grateful to Dr. Bernhard Reinsberg, Lecturer in International Relations, Glasgow University, and Murray Maitland for their continued collaboration and insights throughout the drafting of this paper.

Finally, we would like thank representatives from World Food Programme's Building Blocks, Oxfam's Unblocked Cash, KfW's TruBudget and Seso Global for their time and willingness to share their experiences. 


\section{Table of contents}

Foreword $\quad 4$

Acknowledgements $\quad 5$

$\begin{array}{ll}\text { Executive summary } & 7\end{array}$

1. Blockchain - beyond cryptocurrency to a range of applications 11

Features of blockchain technology 13

$\begin{array}{ll}\text { Proof-of-work versus proof-of-stake } & 15\end{array}$

$\begin{array}{ll}\text { Network types } & 16\end{array}$

$\begin{array}{ll}\text { Debunking common blockchain myths } & 17\end{array}$

2. Blockchain in development co-operation $\quad 19$

Use-case structuring 20

Blockchain for empowerment $\quad 21$

Blockchain for economic development 24

Blockchain for infrastructure and access to services $\quad 28$

3. Four examples of blockchain for development 31

Building blocks - a World Food Programme (WFP) blockchain system for cash transfers 32

Unblocked cash: cash disbursement in Vanuatu $\quad 35$

TruBudget - a KfW initiative 38

Seso Global - a one-stop property shop $\quad 40$

References 43

\section{Tables}

$\begin{array}{lr}\text { Table 1.1. Proof-of-work versus proof-of-stake } & 15\end{array}$

$\begin{array}{lr}\text { Table 1.2. Different types of networks } & 16\end{array}$

\section{Figures}

Figure 1. Policy makers' blockchain decision tree 9

Figure 1.1. Distributed ledgers: centralised, decentralised and distributed $\quad 14$

Figure 2.1. Blockchain for development use-case clustering 20

Figure 2.2. How empowerment-focused blockchain pilots contribute to the SDGs 21

Figure 2.3. How economic development-focused blockchain pilots contribute to the SDGs 24

Figure 2.4. How blockchain pilots improve infrastructure and services to contribute to the SDGs 28 


\section{Executive summary}

\section{The benefits of blockchain for development policy makers}

As the selected case studies and detailed examples in this paper demonstrate, the most profound proven benefits of blockchain support furthering its integration into development activities. These include greater transparency, ability to disrupt established societal models (including ability to alter the balance of power between actors), as well as the potential to greatly reduce inefficient bureaucracy and costs. At the same time, however, the potential trade-offs concerning energy consumption, privacy and scalability, as well as the risk of inequality of access, remain significant. This is compounded by the more fundamental challenges regarding the lack of local skills and infrastructure to support implementation, as well as the lack of international standards agreed by both blockchain and development co-operation providers for applying policy makers to follow.

On balance, this paper posits that policy makers and practitioners in both developed and developing countries must strive to facilitate greater inclusion of this technology. However, in the absence of technical and ethical international standards, this should be done (i) with the aim of contributing to the SDGs and (ii) in alignment with relevant development principles, frameworks and standards. As yet, blockchain remains a junior but significant tool that has the ability to not only support recovery from COVID-19 in the shortterm, but if workable solutions are found for existing energy consumption concerns, it also has the potential to help us 'build forward greener' and ensure planetary resilience over the course of the next 10 to 15 years.

This paper maps development applications of blockchain into three distinct clusters: (i) empowerment (ii) economic development and (iii) improving infrastructure and services before, moving to examine the advantages and drawbacks of four specific examples. Here, key benefits (elaborated in detail in Chapter 2) for policy makers are grouped under each cluster,

\section{Empowerment}

- Blockchain for identification also represents progress towards achieving the SDGs 1, 5, 8, 16 and 17.

- The assertion of self-sovereign identities on blockchain assists those previously "left behind" to access formal societal goods and services.

- Using blockchain to support countries to identify their populations has the potential to equip them with the tools to leverage tax more effectively and foster the growth of the private sector.

- As well as existence, identify provision can also empower previously marginalised individuals to secure proof of ownership, thus combatting institutionalised corruption and strengthening investment opportunities by de-risking the landscape. 


\section{Economic development}

- Blockchain for economic development can help Donors meet commitments to SDGs 1, 2, 3, 5, 8, 9, 10 and 17.

- The transition to digital and cryptocurrencies has the potential to mitigate the worst after effects of the COVID-19 crisis, including inflation.

- By sidestepping traditional financial intermediaries, blockchain can reduce the cost of remittances, an important driver of economic growth across developing countries

- The digitalisation and traceability of processes, for example, allows to better control supply chains, reducing the costs of intermediation as well as linking vulnerable and previously "left behind" populations to the international economy.

- Crosscutting empowerment and economic development, blockchain solutions can improve the efficiency and accountability involved in cash disbursement.

\section{Improving infrastructure and services}

- Blockchain for the improvement of infrastructure and services assist in delivering on SDGs $3,4,7$, 12,13 and 16

- Educational certification on the blockchain can help insure vulnerable populations against attacks or the breakdown of national infrastructure

- Patient control over healthcare can reduce the burden on fragile health systems in developing country contexts

- Blockchain's verified trusted nodes will help to track data and more accurately monitor carbon footprints, upholding important commitments like the Paris Agreement.

Nevertheless, it is important to remember that blockchain remains an emerging, and imperfect, technology. In addition to energy and jurisdictional concerns, when misapplied or implemented without proper due diligence, it can cause significant harm, and even erode stakeholder relationships. It can also be difficult to secure meaningful consent, as well as unintentionally exclude and further destabilise already vulnerable populations. Examples of unintended consequences are plentiful in these scenarios, including potentially deepening the digital divide, refugee data breaches, and the effective creation of two tier societies.

With this in mind, before adopting blockchain solutions, we recommend policy makers consult the decision tree below in Figure 1. 
Figure 1. Policy makers' blockchain decision tree

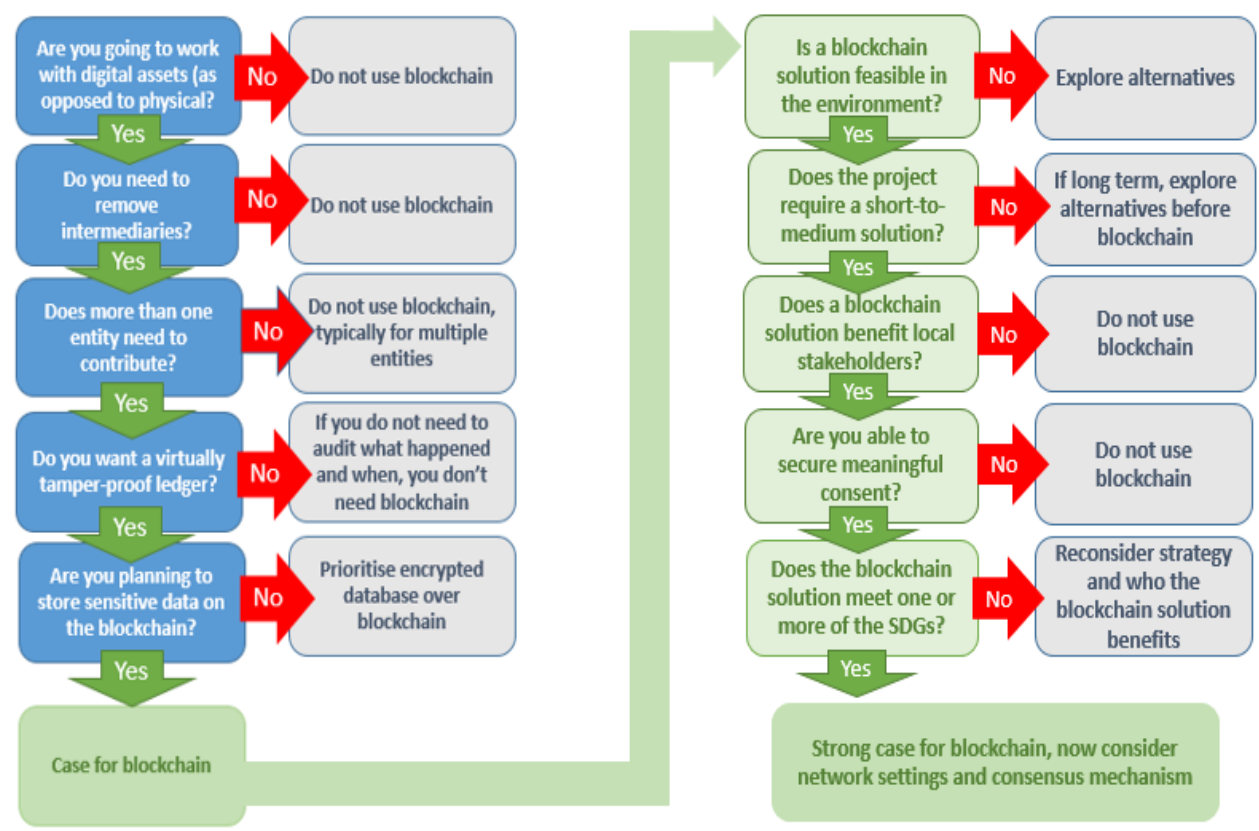

Source: Authors

\section{Recommendations: using blockchain to enhance innovation and produce real development benefits}

Building on both the decision tree pictured above and the analysis contained within this paper we draw preliminary recommendations for policy makers, and public actors more generally, working to implement blockchain solutions:

- Institutionalise knowledge on blockchain technology. To the maximum extent practicable, the public sector should institutionalise knowledge on what blockchain is and how to use it. While policy makers do not need to become technical experts, it is crucial that they have a robust awareness of its use and understand how the technology should be applied in various contexts.

- Look to other contexts for examples of similar successful blockchain pilots before deploying the technology. In other words, avoid experimentation with blockchain solutions in contexts characterised by vulnerable populations. Where possible, solutions should have successful precedents in other contexts before being trialled on local populations in developing country contexts.

- Deploy blockchain only when it brings real benefits. Linked to the point above, blockchain should be viewed as one element in the toolkit of policy responses. Consider who is really set to 'win' from the implementation of a blockchain solution by consulting local governments and stakeholders. Due diligence should include the feasibility of meaningful consent and the exploration of any unintended consequences. 
- Deploy technical assistance alongside blockchain to ensure that local communities have the skills and capacity to assume ownership. Co-designing blockchain solutions with local partners should be undertaken with a view to promoting local ownership and management.

- Ensure compliance with relevant legal and regulatory frameworks. For instance, in public blockchains, consider the law that might apply to the transactions. Likewise, when using private, permissioned blockchains, establish internal regulation and governance control. In as far as possible, incentivise compliance among users.

- Increase collaboration between development agencies and developers of blockchain solutions. In order to harness the full potential of blockchain, it is necessary for development agencies to develop more and better linkages with developers of blockchain solutions. In particular, development agencies must give a chance to the blockchain community to prove what solutions they can offer. This also represents an opportunity for reciprocal learning; blockchain developers can learn what development needs are and how to increase benefits for partner countries and communities.

- Be open to change and innovation. Donors and their development partners should leave room for experimentation of new technologies. Innovation hubs, accelerators and sandboxes must be established with partner governments to explore the use of technologies and facilitate collaboration towards the SDGs.

- Commit to revisiting, reviewing and improving blockchain solutions. Blockchain has the potential to help us meet the SDGs, but remains an emerging technology with relatively few successful pilots in developing contexts. For that reason, we need more and better data. Certainly, policymakers should continuously revisit and re-examine their blockchain-based solutions, sharing case studies and lessons learnt with other agencies where possible. This should be undertaken with a view to continuously adapting to context and stakeholder needs. 


\section{Blockchain - beyond cryptocurrency to a range of applications}

Before analysing potential applications, this first section introduces and outlines blockchain technology, highlighting the most important design features from a development perspective. Features are assessed in terms of their sustainability, accountability, and governance, as well as access and exclusion of vulnerable groups. This section also aims to debunk some of the most common myths that surround the technology and its applications. 
'Blockchain' burst on to the international scene in the wake of the 2008 Global Financial Crisis. Pseudonymous Satoshi Nakamoto first introduced the term in a white paper entitled 'Bitcoin: A Peer-toPeer Electronic Cash System' (Nakamoto, $2009_{[11]}$ ). In response to the trust deficit engendered by the crash, Nakamoto attempted to provoke a discussion around meaningful societal change through the proposal of an alternative form of financing to run on blockchain. The resulting paper outlined a framework for a new type of decentralised digital currency, 'Bitcoin', later termed cryptocurrency.

"Blockchain is to Bitcoin, what the internet is to email. A big electronic system, on top of which you can build applications. Currency is just one." Sally Davies, FT Technology (Tapscott and Tapscott, 2016[12])

Blockchain's trajectory is a testament to the idea that crises can catalyse meaningful change. Conceived in the wake of the Global Financial Crisis, the COVID-19 pandemic has already served to accelerate the further integration of emerging technologies like blockchain. Today, we are witnessing the entry of digital and cryptocurrencies - which use blockchain as their underlying technology - into the mainstream. In the words of Christine Lagarde, President of the European Central Bank, "the coronavirus pandemic has accelerated digital currency adoption [and] this... is unlikely to be reversed once the pandemic is over". (Blockchain News, 2020[13]) While the blockchain landscape is still largely dominated by the United States and Europe, trends like the rise in the use of cryptocurrency in Africa indicates this is set to change. Onlookers note, for example, that a growing number businesses in Kenya are beginning to accept Bitcoin as payment (BBC, 2019[14] ). More generally, the use of mobile money - often facilitated by blockchain technology - has grown significantly in Africa, as the authorities fight to curb the spread of COVID-19 (International Finance, 2020[15]).

The pandemic has also prompted greater reflection on the mainstreaming of blockchain applications beyond the financial and banking sectors. While this paper will discuss the implications of the use of digital and cryptocurrencies, other applications of blockchain technology is one of the principal themes. For instance, the World Economic Forum (WEF) asserts that supply chains powered by blockchain technology could be the key to ensuring the equitable distribution of a COVID-19 vaccine (World Economic Forum, $2020_{[16]}$. Even before the arrival of the pandemic, a diverse array of industries cross-cutting the private and public sectors in both developed and developing countries had begun to experiment with blockchain solutions. These include, but are not limited to, auto manufacturing (Popov, 2020[17]) food traceability (Creydt and Fischer, 2019 ${ }_{[18]}$ ), healthcare (Dwivedi et al., 2019 ${ }_{[19]}$ ), governance (Jun, 2018[20]) and foreign aid (Reinsberg, 2019[21]).

This paper comes at a pivotal moment. While the groundwork for more diverse applications of blockchain has already been laid, the COVID-19 pandemic now urges policy makers to help drive responsible implementation, as part of the generalised shift to a society that is digital by default.

As the selected case studies and detailed examples in this paper demonstrate, the most profound proven benefits of blockchain support furthering its integration into development solutions. These include greater transparency, ability to disrupt established societal models (and linked ability to alter the balance of power between actors), as well as greatly reduce inefficient bureaucracy and costs. At the same time, however, the potential trade-offs concerning energy consumption, privacy and scalability, as well as the risk of inequality of access, remain significant. This is compounded by the more fundamental challenges regarding the lack of local skills and infrastructure to support implementation, as well as the lack of international standards agreed by both blockchain and development co-operation providers for applying policymakers to follow. On balance, this paper posits that policy makers and practitioners in both developed and developing countries must strive to facilitate greater inclusion of this technology. However, in the absence of technical and ethical international standards, this should be done (i) with the aim of contributing to the SDGs and (ii) in alignment with relevant development principles, frameworks and standards. As yet, blockchain remains a junior but significant tool that has the ability to not only support recovery from COVID19 in the short-term, but if workable solutions are found for existing energy consumption concerns, it also 
has the potential to help us 'build forward greener' and ensure planetary resilience over the course of the next $10-15$ years.

\section{Features of blockchain technology}

Readers should note that the technical aspects of blockchain are complex, and as such; this paper does not seek to cover every detail. Rather, the aim is to provide an overall synthesis, underlining the main benefits and potential trade-offs development actors must consider when deciding whether or not to use a blockchain solution, and if so, in its design. We recommend readers consult the OECD Blockchain Primer (OECD, 2018[22]) for more detail on the functioning of the technology. Other useful recommended resources include the USAID Primer on Blockchain (Nelson, 2018[6]) and the GIZ publication, 'Blockchain: A World without Middlemen?' (GIZ Blockchain Lab, 2019[7])

\section{What is blockchain?}

"Currently, most people use a trusted middleman such as a bank to make a transaction. But blockchain networks allow consumers and suppliers to connect directly, removing the need for a third party" (Hutt, 2016[23])

Put simply, blockchain is a cryptographically linked database or ledger, comprised of chains of data blocks. A 'ledger' is comparable to a record book in that it time-stamps, records and stores all transactions between users in chronological order. (Rosic, 2016[24])

Another helpful way of understanding how the technology works is Daley's 'Google Doc' analogy. While blockchain is much more technically complex than a Google Doc, there are some useful similarities. That is, an individual distributes a document with a group when using Google Doc, as opposed to replicating or transferring copies. The fact that everyone can connect to the document at the same time and no one is excluded from the document while changes occur makes this a decentralised delivery chain. Further to this, the real-time nature of the edits renders the whole process highly transparent. (Builtin, 2020[25])

\section{The three distinctive features of blockchain}

While the specificities differ according to permission setting, several characteristics are common to all blockchains. In general, the blockchain is (i) distributed, (ii) immutable, and changes require (iii) agreement by consensus.

Unlike traditional, centralised ledgers, the blockchain ledger is distributed across a network of computers (Figure 1.1), building resilience against attacks. Nodes are computers connected to the blockchain network (ConsenSys, 2020[26]). As a result, each trusted node ${ }^{1}$ in the blockchain network retains an identical copy of the existing blockchain.

In order to synchronise the data, the trusted nodes process each block (group of transactions), determining its validity. A final conclusion is determined using a consensus mechanism (detailed below).

\footnotetext{
${ }^{1}$ There is no single type of blockchain, and the number of trusted nodes with the ability to act as an intermediary or authority is subject to network type, which will be explained in more detail later in this chapter.
} 


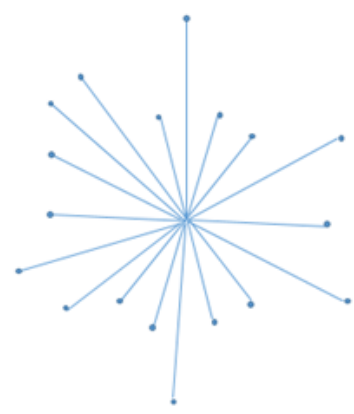

Centralised

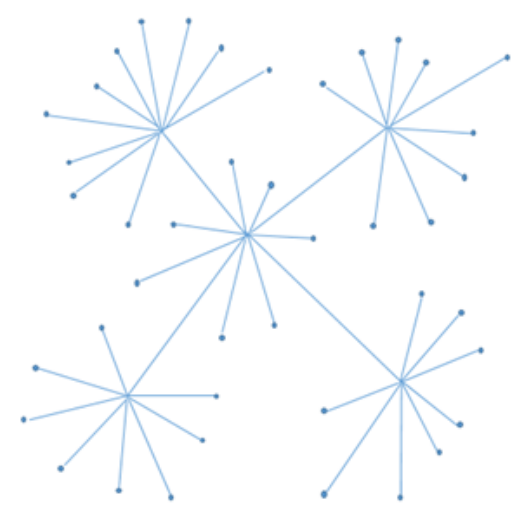

Decentralised

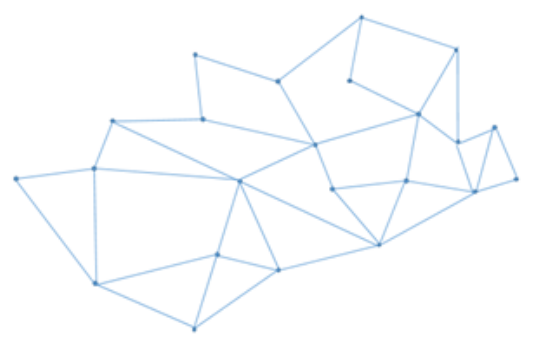

Distributed

Source: Authors

Due to its distributed nature, blockchain has enabled "trust-less interactions among individuals without the need for central authority" (Christie, 2020[27]). Readers should note that in reality trust previously invested in intermediaries does not disappear, but is rather reassigned to the coders behind individual blockchain design. However, for the purposes of this explanation, this 'trust-less' assertion rests on the fact that - in open blockchains at least - all nodes are trusted have access to an immutable transaction record. In the vast majority of cases, transactions cannot be modified after initial creation. The broadcasting of timestamped blocks to nodes prevents double spending. This immutability is secured through a cryptographic hashing algorithm. ${ }^{2}$

The tamper-resistant nature of blockchain is perceived as one of its key advantages vis-à-vis traditional, centralised ledgers.

Final agreement on data is achieved via automated consensus mechanisms. This is a process common to all blockchains, where 'miners' pick up information from nodes and perform a special process called 'mining' on it. To validate the data, miners compile the transaction data to create a hash. Subsequent hashes will contain the original hash plus new transaction data (ICO.li, 2019[28]). Updating the ledger with additional information is a collective process, achieved via consensus. (Christie, 2020[27]). Different consensus models exist. For instance, the Ethereum and Bitcoin blockchains operate using a model called proof-of-work (POW), which incentivises nodes to compete against each other to broadcast blocks for approval. If successful, they are rewarded in Bitcoin (OECD, 2018[22]).

\footnotetext{
${ }^{2} \mathrm{~A}$ hash is like a digital fingerprint; it is unique to each piece of data on the blockchain. The process of hashing refers to having an input item of a certain length, which corresponds to an output item of a fixed length. (Rosic, 2017[111]) In other words, the specific input, if unchanged, will always produce the same exact hash.
} 


\section{Proof-of-work versus proof-of-stake}

The type of consensus mechanism used will influence to what extent the blockchain-based intervention can truly meet the development goal. Table 1.1 below depicts the main benefits and drawbacks of two key commonly used consensus mechanisms.

\section{Table 1.1. Proof-of-work versus proof-of-stake}

\begin{tabular}{l|l}
\hline \multicolumn{1}{c|}{ Proof-of-work (PoW) } & \multicolumn{1}{c}{ Proof-of-stake (PoS) } \\
\hline $\begin{array}{l}\text { Miners invest in equipment and use energy resources } \\
\text { to increase the chance of winning the race to validate } \\
\text { blocks. }\end{array}$ & $\begin{array}{l}\text { Users invest in 'stakes' as collateral. The algorithm } \\
\text { chooses the block creator based on the 'stake'. }\end{array}$ \\
\hline $\begin{array}{l}\text { Malicious activity is possible but only with a computer } \\
\text { more powerful than the majority }(51 \%)^{3} \text { of the } \\
\text { network. }\end{array}$ & $\begin{array}{l}\text { Malicious activity much harder, as you would have to } \\
\text { own } 51 \% \text { of the all the cryptocurrency in the network. }\end{array}$ \\
\hline $\begin{array}{l}\text { The first miner to solve the puzzle and validate is } \\
\text { rewarded, usually in cryptocurrency like Bitcoin. }\end{array}$ & $\begin{array}{l}\text { Block creator creates a transaction fee as no } \\
\text { automatic reward. }\end{array}$ \\
\hline
\end{tabular}

Source: Authors, adapted from (Bitdegree, 2018[29]) 'Proof-of-Work VS Proof-of-Stake: Which One Is Better?' https://www.bitdegree.org/crypto/tutorials/proof-of-work-vs-proof-of-stake

The total electricity consumption of Bitcoin, which uses PoW, was estimated to be more than Austria's national consumption in 2015 (Denis Le Sève, Mason and Nassiry, 2018[30]). The high consumption of energy PoW involves renders it largely unsuitable for development actors, who are bound by the Paris Agreement to promote sustainability (United Nations Framework Convention on Climate Change, 2016 [31]) and the UN Global Compact, in which Principle 9 calls on development actors to "encourage the development and diffusion of environmentally friendly technologies" (United Nations Global Compact, $2004_{[32]}$ ). A further energy-related drawback of this consensus mechanism is it also risks excluding the primary targets of development co-operation work. This is due to the fact that under the PoW consensus mechanism, those with the fastest computers and broadband are much more likely to receive rewards for first validation. In practice therefore, the slower computational power of developing countries (in 2016, $14 \%$ of individuals in low-income countries had internet access compared to $82 \%$ in high-income countries (Denis Le Sève, Mason and Nassiry, 2018[30])) effectively excludes their citizens from participating in blockchain PoW consensus as miners, therefore potentially indirectly exacerbating the digital divide.

Again, as indicated above, PoS users on the other hand make an investment in the token itself as opposed to the equipment and electricity. The major benefit of PoS from a development perspective is that it eliminates the energy consumption dilemma. Further benefits include the reduced risk of malicious behaviour on the chain, such as ' $51 \%$ attacks'. On the other hand, experts note that the more complicated nature of the algorithm and minimum staking requirement can serve to exclude the average investor from participating.

It is worth emphasising that all consensus mechanisms have advantages and drawbacks. Therefore, development actors must consider the relative merits of each in terms of the local development context when designing a blockchain solution. Readers should also note that a growing number of alternative consensus mechanisms are emerging, such as the proof-of-authority. PoA lets trusted users manipulate the blockchain, and has been used both by the WFP (as will be elaborated in Chapter 3 ) and the World Bank (Reinsberg, 2020[33]).

\footnotetext{
${ }^{3} 51 \%$ attacks: If nodes collude together and manage to control more than $50 \%$ of the computational power in the network, they can dictate which blocks to add to the ledger. If successful, they can erase or double-count old blocks transactions - without anyone else in the network discovering it (Bitdegree, 2018[29]
} 


\section{Network types}

As well as variation in consensus mechanism, blockchains differ according to the type of network - or rather, permission models - used. As aforementioned, blockchain remains an emerging technology. Relative youth, coupled with the speed of blockchain's rise, has led to inconsistent treatment and definition of key terminology. However, for the purposes of this paper, we will identify four basic types of blockchain network, identified in Table 1.2 below.

\section{Table 1.2. Different types of networks}

\begin{tabular}{|c|c|c|c|c|c|}
\hline & & Who can view? & Who can edit? & $\begin{array}{c}\text { Who can update the } \\
\text { status? }\end{array}$ & Example \\
\hline \multirow{2}{*}{ Open } & $\begin{array}{c}\text { Public, } \\
\text { permissionless }\end{array}$ & Anyone & Anyone & Anyone & $\begin{array}{l}\text { Bitcoin, } \\
\text { Ethereum }\end{array}$ \\
\hline & $\begin{array}{c}\text { Public, } \\
\text { permissioned }\end{array}$ & Anyone & Only authorised & All or only authorised & $\mathrm{NeO}$ \\
\hline \multirow{2}{*}{ Closed } & Consortium & Only authorised & Only authorised & All or only authorised & Hyperledger \\
\hline & Enterprise & $\begin{array}{l}\text { Participant(s) from } \\
\text { single organisation }\end{array}$ & $\begin{array}{l}\text { Fully private or } \\
\text { restricted to } \\
\text { authorised }\end{array}$ & Network operator & $\begin{array}{c}\text { Quorum (from JPMorgan } \\
\text { Chase) }\end{array}$ \\
\hline
\end{tabular}

Source: Authors

\section{Public, permissionless network (open)}

As indicated above, a public, permissionless blockchain (such as Bitcoin or Ethereum) is a form of open network, where anyone can download the protocol and read, write and update the status. Each new block is timestamped and validated by all nodes before it is written into the blockchain. Importantly, all transactions are public, and all nodes are equal. Public blockchains are effectively immutable, as the data cannot be altered.

Public, permissionless networks arguably represent blockchain's real revolutionary power; no one entity has absolute control over the network and anyone can participate (Joudrey, 2019[34]). However, because each node is required to validate each transaction, these networks tend to suffer from low transaction throughput ${ }^{4}$ and thus cannot be scaled easily. For instance, Bitcoin has a peak capacity of around eight transactions per second, compared to approximately 56000 for Visa's network (Mulligan, 2016[9])

\section{Public, permissioned networks (open)}

A hybrid between open and closed networks, public, permissioned networks allow anyone to read the ledger, but only a select number of authorised participants are allowed to contribute to the ledger. This type of network allows multiple customisation options, such as permitting individuals to join after confirmation of identity, and allocation of select permission. Supply chains may find this type of network

\footnotetext{
${ }^{4}$ Throughput: the rate at which valid transactions are committed in a defined period
} 
useful in order to 'solve' perceived issues related to transparency and auditability; the public can view the information but only authorised employees can write and update the ledger (OECD, 2018[22])

\section{Private, permissioned networks (closed)}

Private, permissioned blockchains are closed networks which limit the openness of data by design. They achieve this by protecting transaction data from third parties, and often a participant is invited to join through an authentic and verified process. Unlike the democratic characteristics of a public blockchain, in private, permissioned blockchains, only select network operators (in Consortiums this can be more than one individual) have the rights to override, edit or delete the necessary entries on the blockchain as required.

Private, permissioned blockchains are more appropriate for organisations bound by more stringent confidentiality clauses, or organisations obliged to protect the data of potentially vulnerable beneficiaries and clients. It is also relatively more simple to devise a legal framework and internal governance structure (Salmon and Myers, 2019 ${ }_{[35]}$ ). Further, they are also faster and more scalable than their open counterparts due to the more centralised structure involving fewer nodes to run the consensus mechanism. Practically, this means private networks can support and process comparatively higher transactions. However, the presence of fewer 'trusted nodes' can also render private networks susceptible to hacking and the manipulation of data.

Altogether, the lack of openness indicates that the technology is still very much in its infancy and important barriers remain. As this section has demonstrated, a multiplicity of blockchains, each with different characteristics exist. This points to lack of interoperability, one of the most fundamental barriers to widespread implementation of blockchain-based solutions. Interoperability refers to different blockchain networks' ability to interact and 'speak' to each other (Finextra, 2020[36]). More specifically, though public blockchains hold the real potential to transform trust-less environments and the relationship between actors, from a development perspective, they lack satisfactory solutions to key issues like scalability, energy consumption, and data protection for vulnerable groups. The result must be careful consideration between policymakers and their development partners when designing the base protocol, particularly over decentralisation, security, and throughput.

\section{Debunking common blockchain myths}

One of the main themes of this paper is to debunk the most pervasive blockchain misunderstandings amongst development co-operation actors - that the use of the technology is restricted to cryptocurrencies. Here, we address some other common blockchain myths.

\section{Is blockchain un-hackable?}

The perceived immutability of data, or, in other words, the inability to hack a blockchain, is often heralded as one of its key transformative benefits.

However, in reality, a blockchain's specific level of security is dependent upon the consensus mechanism utilised, and corresponding network setting. For instance, the most common consensus mechanism, PoW, can be subject to malicious $51 \%$ attacks. However, the threat of a $51 \%$ attack can be mitigated by the size of the network; in large networks such as Bitcoin and Ethereum, it is practically impossible for one node to possess sufficient computational power to execute a profitable $51 \%$ attack. Nevertheless, as recently as January 2019 a 51\% attack was detected on the Ethereum Classic blockchain, proving that blockchains are not fully immutable (Jenkinson, $\left.2019_{[37]}\right)$. 


\section{Are blockchain transactions untraceable?}

One of the main misconceptions is that blockchain transactions are anonymous. In reality, perhaps a better way to describe blockchain activity is pseudonymous (Ledger Ops, 2019 $9_{[38]}$ ). For example, although a user's private information is hidden from all other users in a public blockchain like Bitcoin, the address of the node involved in a transaction is visible. This long string of numbers and letters does not contain any identifiable information that would tie the user to the address or its associated wallet (Ledger Ops, 2019 [38]). So while it is possible to trace the history of that owner's transactions with the address, to go a step further and match an address to an actual beneficial owner identity is near impossible, unless the user exposes themselves willingly by giving up their private key.

While the above is true for public, permissionless blockchains, their private counterparts can, in theory, serve to decrease the ability of external third parties to trace transactions. While the network requirements to join a network are much more stringent, requiring greater levels of information, it is possible to anonymise an address to the extent that it is only visible to direct transaction partners.

\section{Are blockchains associated with illicit activities?}

Arguably, blockchain became best known over the past decade through the association of cryptocurrencies like Bitcoin with illicit activity. This association was seemingly cemented following the United States' Federal Bureau of Investigation's shutdown of the online black market Silk Road in 2013 (United Nations Economic and Social Commission for Asia and the Pacific, 2017 $\left.{ }_{[39]}\right)$. Certainly, the pseudonymous nature of blockchain renders certain applications, namely cryptocurrencies, convenient vehicles to engage in illicit trade and suspicious activities, like drug trafficking, hacking and threats. Reports even suggest that in the last quarter of 2019, the amount of cryptocurrency spent on the dark net grew $60 \%$ to USD 601 million (New York Times, 2020[40]).

It is important to underscore that any technological developments often unintentionally promote criminal activity. For example, the United Nations' Office on Drugs and Crime (UNODC) reports that the internet, whilst providing a platform for people to sell legitimate goods, also allows "criminals to sell drugs, firearms and endangered wildlife" (United Nations Office on Drugs and Crime, 2017[41]). Nevertheless, Bitcoin's bad reputation, and the conflation of Bitcoin and blockchain, has left many countries wary. India is a prime example, where the legalisation of Bitcoin is a "hotly-contested policy issue between the Ministry of Finance, which would like to tax it, and the Reserve Bank of India, which has declared bitcoin illegal and in breach of anti-money laundering provisions" (IFC, 2017[42]).

As yet, there are no concrete provisions that adhere to anti-money laundering laws (AML) to monitor any suspicious patterns. Further to this, the decentralised nature of blockchain, which spans several entities across locations, gives rise to significant jurisdictional challenges (Salmon and Myers, 2019[35]). This in turn increases the risk of money-laundering and terrorist financing. A co-ordinated multilateral response, building on the Guidance for a Risk-Based Approach to Virtual Assets and Virtual-Asset Service Providers, announced by the Finance Action Task Force in June 2019 (Financial Action Task Force, 2019[43]), will be necessary going forward to develop and promote regulation. 


\section{Blockchain in development co- operation}

The applications of blockchain have expanded beyond cryptocurrencies to a broad suite of sectors, including foreign aid and development cooperation more generally. The primary objective of this chapter is to map existing use-cases before narrowing the lens to more detailed analysis of blockchain in development co-operation through four specific examples. The mapping primarily focuses on use-cases piloted in developing countries, but due to the aforementioned small number of use-cases, a select number of use cases from high-income countries are also examined. These case studies were selected on the basis of the extent to which they demonstrate the potential for blockchain to respond to similar needs in developing countries. 


\section{Use-case structuring}

For the purposes of this paper we have clustered pilots into the following sub categories (also visualised in Figure 2.1) in order to help guide policy makers and their development partners:

- Empowerment (such as identity provision - including for refugees - as well as land rights and transparent voting procedures);

- Economic development (including digital and cryptocurrencies, as well as remittances and supply chains) and

- Improving infrastructure and services (including healthcare and education, as well as towards clean energy markets).

Figure 2.1. Blockchain for development use-case clustering

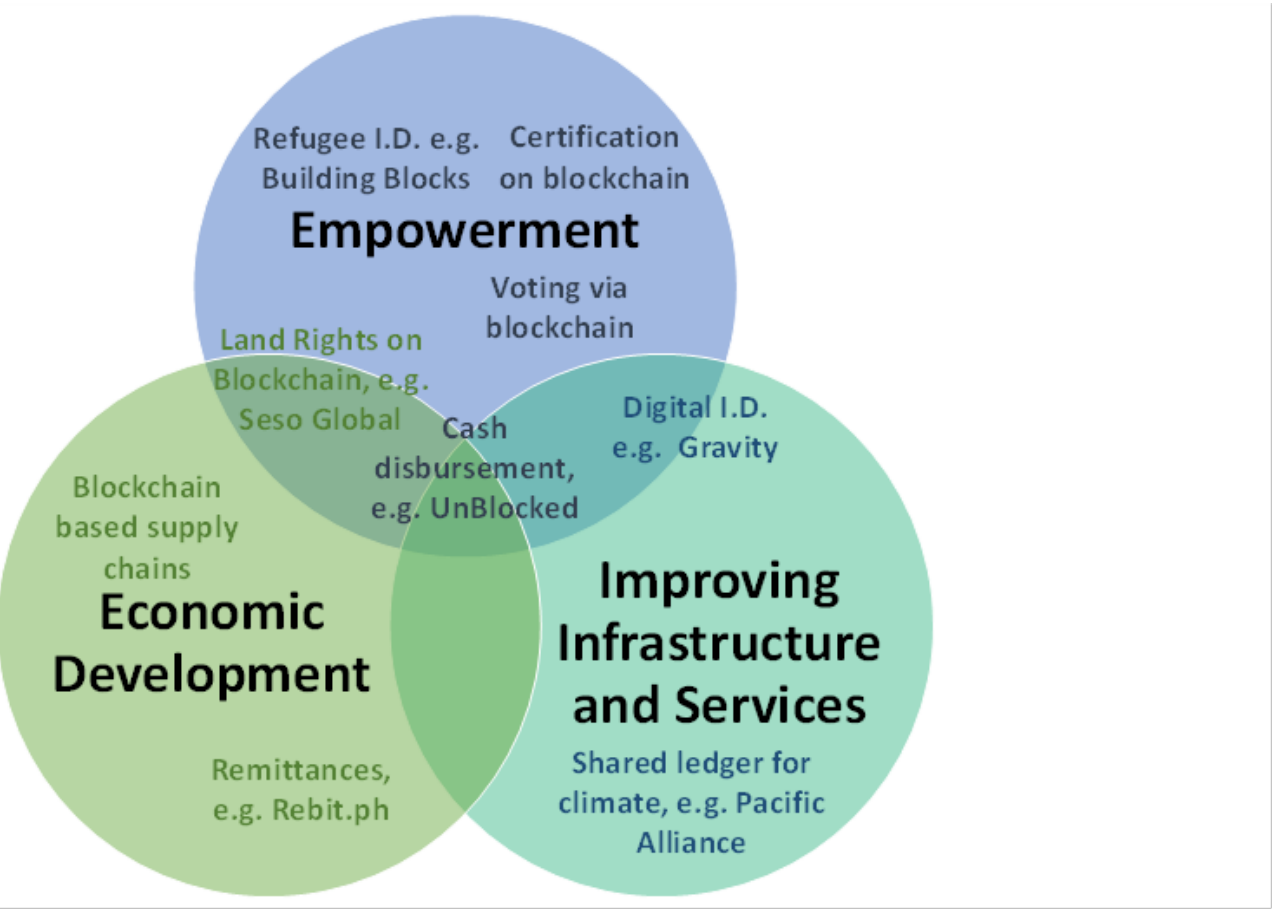

Note: Examples have been provided where pilots in developing country contexts already exist, as well as potential areas where blockchain may be a useful tool in responding to particular development needs

Source: Authors

Within each of these sub-groups, select examples detail the potential transformative benefits and draw backs of using blockchain in development co-operation. Where relevant, analysis will indicate how each of the selected examples contribute to the realisation of the Sustainable Development Goals (SDGs) and the 2030 Agenda. While there are encouraging signs towards the adoption of regulation on user protection, as highlighted by the Global Digital Finance Code (Global Digital Finance, 2019[44])and the Presidio Principles (World Economic Forum, 2020[45]), internationally agreed technical and ethical standards for blockchain implementation do not yet exist. As such, this paper proposes development principles, frameworks and standards as a guide for policymakers and their development partners where relevant. This exercise aims to promote the discriminate and responsible implementation of the technology by policy makers and their development partners. 


\section{Blockchain for empowerment}

This sub-section examines how blockchain pilots clustered under 'empowerment' can contribute the SDGs, as visualised in Figure 2.2 below.

\section{Figure 2.2. How empowerment-focused blockchain pilots contribute to the SDGs}
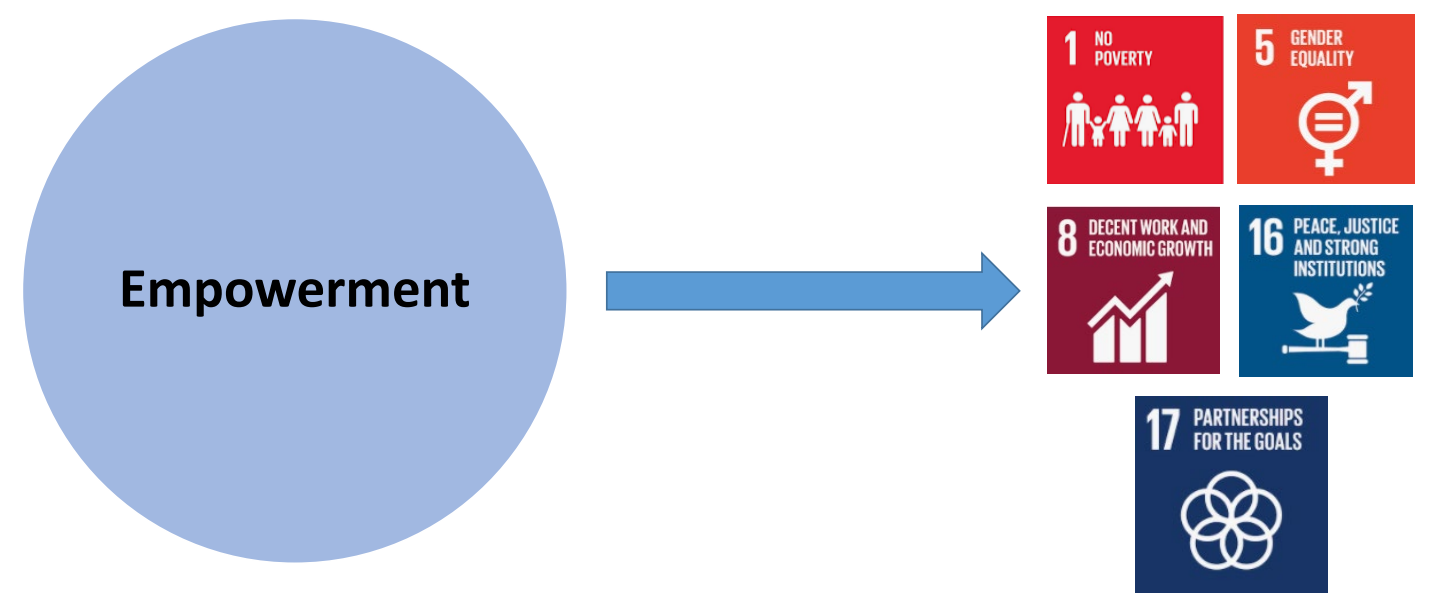

Source: Authors

Effective identification programmes are crucial for development, and are enshrined in the SDGs. SDG Goal 16.9, for example, under 'Peace, Justice and Strong Institutions', calls on countries to, "by 2030, provide legal identity for all". This Goal also features birth registration indicator 16.9.1, which examines the "proportion of children under five years of age whose births have been registered with a civil authority" (United Nations, 2015[46]). Likewise, indicator 17.19.2 under 'Partnerships for the Goals', determines the "proportion of countries that have (a) conducted at least one population and housing census in the last ten years; and (b) have achieved $100 \%$ birth registration and 80\% death registration" (United Nations, 2015[46]). Beyond the SDGs, the Universal Declaration on Human Rights stipulates that "everyone has the right to recognition everywhere as a person before the law" (United Nations, 1948[47]).

Figures vary, but estimations place the number of individuals who currently lack official identity between one and two billion worldwide (Coinfy, 2017[8]). At an individual level, people without recognised identification can struggle to access finance and formal societal participatory activities, such as school enrolment. Typically, of those individuals affected, a disproportionate number are women and children, as well as poor and marginalised communities, including refugees. As such, by working on blockchain projects to empower individuals in developing countries with official identification, states can progress towards fulfilling their 2030 Agenda pledge to "ensure no one is left behind" (United Nations, 2015[46]). More specifically, as women are among those most likely to be affected by lack of identification, pilots in this area offer policy makers the opportunity to contribute to SDG 5 'Gender Equality' (United Nations, 2015[46]). 
More broadly, official identification of the population is also key to a healthy, functioning economy (ConsenSys, 2020[48]). For instance, those countries without the ability to identify large segments of the population may be less equipped to raise tax and foster growth within their private sectors (World Bank, 2018[49]). The constrained ability of developing countries to raise domestic resources assumes renewed significance in the wake of the COVID-19 crisis, as inflows of external private finance are projected to drop by as much as USD 700 billion compared to 2019 , exceeding the impact of the 2008 crash by $60 \%$. (OECD, $2020_{[50]}$ ) This will also serve to compound the SDG financing gap in developing countries, which stands at USD 2.5 trillion, and existed well before the COVID-19 crisis struck (OECD, 2020[50]). Again, this indicates that a potential indirect consequence of working to promote more systematic official identification in developing countries is the contribution to both SDG 1, 'No Poverty' and SDG 8, which calls for 'Sustained, Inclusive and Economic Growth' (United Nations, 2015[46]).

There are a number of successful pilot initiatives in developing countries that demonstrate early examples of blockchain-based solutions in specific contexts used in order to empower individuals and help assert their self-sovereign identity without the necessity of relying on a potentially untrustworthy intermediary. One use-case is Gravity, which ran a first pilot in Kenya, registering 1000 users in just three days. The initiative helps individuals establish a trusted identity of proof of existence independent from public infrastructures and non-dependent on traditional official identity documents, postal addresses or banking systems (GSMA, 2017 ${ }_{[51]}$ ). It achieves this by enabling the customer-declared information to go through a process of distributed vetting. The virtual impossibility of manipulating information stored on the blockchain without detection serves to enhance trust amongst all users.

Another interesting pilot in this domain is BanQu, which creates economic identities for smallholder farmers. BanQu's mission as a blockchain-based platform is to connect the some 2.3 billion people who depend on agriculture for their livelihoods, to the global economy. This is achieved via the provision of a secure, portable digital identity. (GSMA, 2017 ${ }_{[51]}$ ) With BanQu, anyone with a phone is able to connect to the network and set up their personal economic identity. It is worth underlining that such initiatives rely on the increasing rate of mobile phone penetration in developing countries. For instance, recent studies highlight that two thirds of the world's population had a mobile phone in 2017, and by the end of 2020 the global penetration rate is predicted to reach 75\% (GSMA, 2017[51]). The BanQu business model encourages the user to begin to accumulate a history of their transactions and assets on the BanQu blockchain. In turn, this starts to build a traceable economic identity, which can be used to access infrastructure and services. Such platforms are gaining increasing traction in the wake of COVID-19. For example, companies like Zambian Breweries have praised the BanQu platform for facilitating continued payments to cassava farmers during the pandemic (BanQu, 2020[52]).

One of the groups most likely to be affected by lack of formal documentation are refugees; documents are often lost or scattered in the process of an intense and often unexpected journey. Even where this does not apply, home countries cannot necessarily be relied upon to provide this data. Altogether, the Norwegian Refugee Council notes that $70 \%$ of Syrian refugees lacked basic ID (Aburass, $2017_{[53]}$ ). As will be explored in greater detail in the following chapter, The World Food Programme (WFP)'s Building Blocks employs blockchain for biometric identification, aiding in the efficient provision of goods and services to refugees. Similarly, Finland has piloted the use of pre-authorized digital cards for refugees that are linked to the blockchain, thus linking financial resources to identification (Rayner, 2018[54]).

In many ways, the refugee example crystallises the multiple benefits and drawbacks of using blockchain for empowerment. On one hand, the increased autonomy for refugees associated with blockchain-based solutions like Building Blocks puts the 'human' back in humanitarian aid disbursement. Nevertheless, the risk of potential data infringement is a significant and as yet under-explored danger (Christie, 2020[27]). Such an incident, if it were to take place, runs the risk of further persecution and discrimination of already vulnerable communities, exemplified by the Rohingya refugees in Bangladesh (Christie, 2020[27]). We therefore 
Recommend that if DAC donors intend to develop blockchain-based identity solutions, they should consider the advantages offered by private, permissioned blockchains first and foremost.

Closely linked to blockchain's ability to empower individuals to assert their own sovereign identities, is the technology's strength in improving governance mechanisms and mitigating corruption. Corruption, both state-sponsored and external, can take many forms, and creates barriers to access. It is noteworthy that $70 \%$ of people, particularly in certain African countries characterised by a lack of legacy systems, do not have legally registered titles to their land. (Coinfy, $2017_{[8]}$ ). Notably, women, the poor, marginalised groups and indigenous peoples are most vulnerable to arbitrary practices in land governance. (GIZ Blockchain Lab, $\left.2019_{[7]}\right)$ As a tamper-resistant, time-stamped ledger, blockchain is a useful tool when it comes to recording accurate data on the chain, in this case certifying proof of ownership and existence. Several frequently cited pilots involving blockchain for land registration include Bitfury in Georgia (Institute of Development Studies, 2017[55]) and the Indian state of Andhra Pradesh, which is exploring the use of blockchain in this context. (Coinfy, 2017 ${ }_{[8]}$ ) Land documentation will be analysed in more detail under the Seso Global use-case in the following chapter, including the risks of using an emerging technology for long-term development projects.

While the literature review did not reveal a developing country blockchain pilot linked to voting, it seems that Estonia's experience as the first country in the world to pilot online voting elections (Sayers, 2017[56]) could be replicated in developing country contexts stymied by a lack of trust in traditional intermediaries. Altogether, blockchain can positively "impact democratic voting models and promote the principles of good governance" (BlockXLabs, 2018[57]). Certainly, tracing votes to valid and registered identities stored on a blockchain reinforces democratic elections, as the digital casting of votes is granted to registered citizens only and cannot be double-counted by a third party, such as a public electoral authority.

In empowering communities to claim their identities, blockchain technology can assist donors and policymakers in fulfilling their commitments to 'leave no one behind' and make tangible inroads towards SDGs $1,5,8,16$ and 17. This is in part due to the fact the technology can help reduce information asymmetries between donors and ultimate beneficiaries (Reinsberg, 2019[21]). However, this brief overview indicates that the multiplicity of new factors involved in blockchain projects underlines the necessity for sound understanding of local needs to a greater extent than ever before. Notably, this is mandated by the Busan Principles on development effectiveness, which stipulate that "partnerships for developing countries can only succeed if they are [...] tailored to country specific situations and needs" (OECD, 2011[58]). Similarly, the OECD's Principles for Managing Sustainable Development Results specify the need to "adapt to context". (OECD, 2019 $\left.{ }_{[59]}\right)$ Carrying out blockchain solutions with care is crucial to building robust and sustainable stakeholder relationships (Christie, 2020[27]). As such,

Donors should provide sufficient funds for technical assistance to ensure beneficiaries are aware of the risks entailed and can offer meaningful consent.

Perhaps most importantly, it is

Imperative that donors ensure the implementation of digital technologies like blockchain do not unintentionally
exacerbate existing social inequalities, by, for instance, unintentionally contributing to the creation of a two-tier
identification system.

The pilots outlined in this section provide identification for specific purposes, and it is as yet unclear how robust they would be if challenged in court. As such, in the short-term, donors should be wary of viewing blockchain-based identity as a wholesale replacement for formal identification. Rather, solutions should be deployed to solve specific identity problems in development contexts. At the same time,

Donors should also continue to invest in and support traditional formal identification systems, until such a moment when it might be possible to digitise national infrastructure. 


\section{Blockchain for economic development}

Technological innovation is often regarded as a necessary component in the drive towards greater economic growth and development. With the 'Decade of Delivery' firmly underway and the pace of change faster than ever, the potential for emerging technologies such as blockchain to spur economic development and help us meet the SDGs (Figure 2.3) has never been more important. Conscious of the many areas that fall under the broad umbrella of economic development readers should note this section focuses on digital and cryptocurrencies, remittances, supply chains and aid disbursement only.

\section{Figure 2.3. How economic development-focused blockchain pilots contribute to the SDGs}
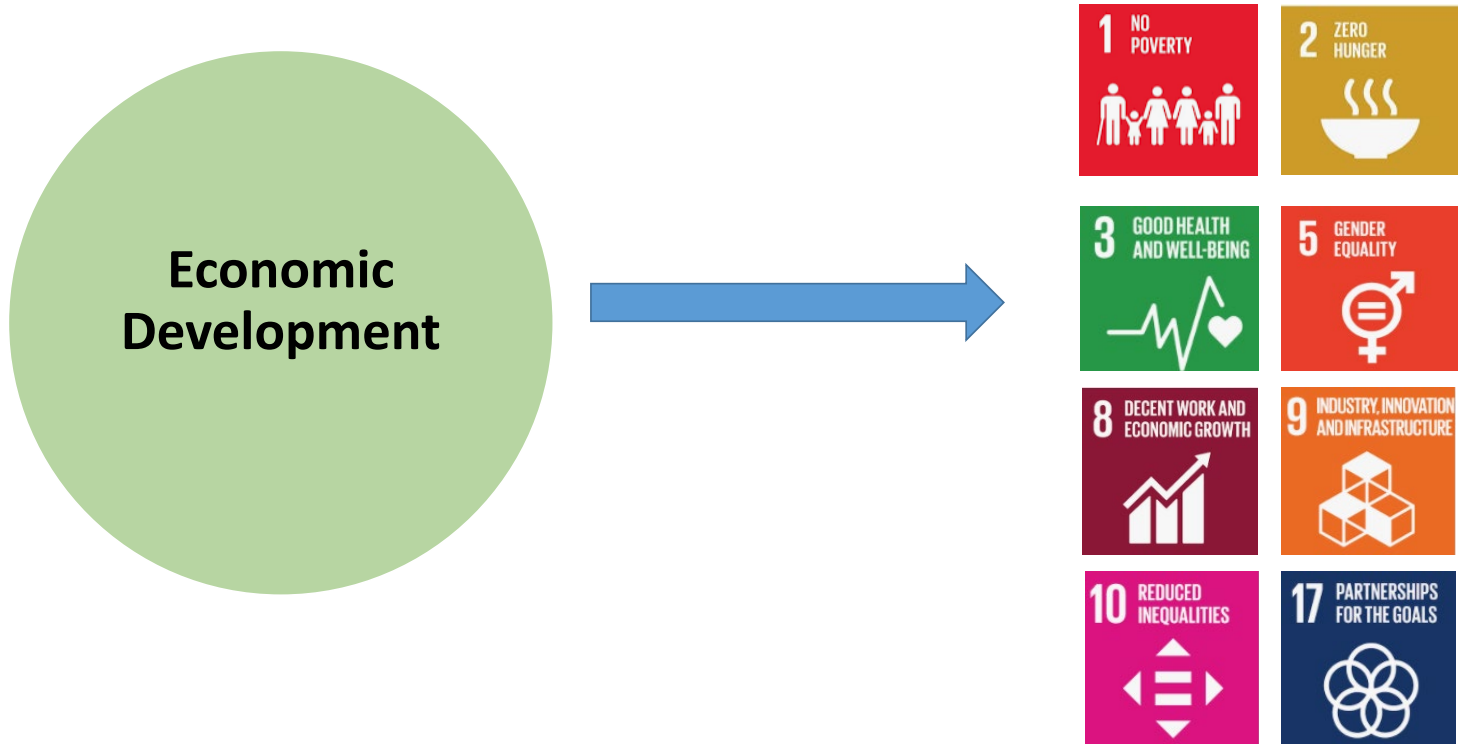

Source: Authors

One of the most exciting potential uses of digital and cryptocurrencies powered by blockchain is their ability to promote financial inclusion (United Nations Economic and Social Commission for Asia and the Pacific, 2017 [39]), an explicit target of seven of the 17 SDGs. These include SDG 1 'No Poverty'; SDG 2 'Zero Hunger'; SDG 3 'Good Health and Well-Being'; SDG 5 'Gender Equality'; SDG 8 'Decent Work and Economic Growth'; SDG 9 'Industry, Innovation and Infrastructure', and SDG 10 'Reduced Inequalities' (United Nations, 2015[46]). The United Nations Capital Fund (UNCDF) also asserts that there is a case for SDG 17 'Partnerships for the Goals', which they argue contains "an implicit role for greater financial inclusion through greater saving mobilisation for investment and consumption that can spur growth" (United Nations Capital Development Fund, 2020[60]).

Many developing countries represent fertile environments for blockchain-based financial solutions. For instance, sub-Saharan Africa is home to many unbanked individuals (a high proportion of whom are women and other marginalised demographics) and the lack of legacy systems more generally remove the element of cultural resistance experienced in developed contexts. Notably, $70 \%$ of transactions in Kenya are already digital (IFC, 2017[42]), and the results of a long-term study on M-Pesa, Kenya's mobile money service, found mobile money had contributed to lifting 194000 households (equivalent to $2 \%$ of the population) out of poverty (United Nations Capital Development Fund, 2020[60]). Elsewhere in the 
Continent, South Africa is well known for its friendly FinTech regulatory environment, and is home to numerous blockchain-based financial start-ups, including GeoPay and Bitsure (IFC, 2017 ${ }_{[42]}$ ). Likewise, in January 2020, the Senegalese government finalised a deal with Senegalese-American singer Akon, to build a cryptocurrency-based city using the Stellar (XLM) network in the country (Forbes, 2020[61]).

Similarly to blockchain-based identification solutions, in the context of existing national infrastructure, the use of cryptocurrencies risks the unintentional creation of a second-tier banking system. A worst-case scenario entails a situation where those "left behind" would be locked out of the existing formal banking system where they could theoretically, eventually obtain access to credit. However, projects like Akon's point to the idea that

Cryptocurrencies, when supported with the right infrastructure, are not just a next-best option for these communities. Rather, they have the potential to lead to deeper financial inclusion across society.

Without doubt, COVID-19 has rendered innovative blockchain projects like Akon's, which provide economic development by promoting financial inclusion, more important than ever (Forbes, 2020[61]). A further advantage for developing countries faced with escalating crises in the wake of COVID-19, is that cryptocurrencies protect against the worst excesses of inflation; "the supply of cryptocurrencies is fixed, meaning that they cannot be manipulated through money printing" (Toolbox, 2020[62]) However, major draw backs include volatility, stemming from the decentralised nature of blockchain (United Nations Economic and Social Commission for Asia and the Pacific, 2017 ${ }_{[39]}$ ), as well as the well-known risks entailed in using public networks, such as the necessity of high computational power and potential exposure of sensitive data to malicious or illicit activity.

Another important element of digital and cryptocurrencies is remittances. The OECD defines remittances as "financial or in-kind transfers made by migrants to friends and relatives back in their countries of origin" (Rühmann, 2020[63]). Remittances are highly beneficial to low and middle-income countries, and are considered a relatively stable source of foreign exchange. A record USD 529 billion in remittances flowed to low and middle-income countries in 2018, making remittances three times higher than official development assistance (ODA) (Rühmann, 2020[63]). Given the volume of these flows, remittances promote growth in less financially developed countries as an alternate source of investment finance. However, up until now, the highly fragmented and complex nature of the traditional global banking system has resulted in high costs (estimated to be $6.9 \%$ per USD 200) associated with sending remittances (Rühmann, 2020[63]).

Recognising the importance of remittances for global economic development, several pilots are harnessing blockchain technology to lower the cost of international payments. Cryptocurrencies like Bitcoin have the power to sidestep costs of international payment settlements by ensuring that all transactions take place either within a national payments system or over the digital currencies' blockchain network.

Where countries have already established digital currency exchange markets, the results from using blockchain technology can prove effective.

For example, sending USD 200 remittance from the United States to the Philippines with Rebit.ph costs $3 \%$, much nearer to SDG target indicator 10c, which aims to reduce the cost of remittances to less than $3 \%$ (United Nations, 2019[64]). Lastly, it is worth underlining that, with remittances, users can buy and spend cryptocurrency within minutes, greatly reducing price volatility concerns associated with cryptocurrency more generally. (United Nations Economic and Social Commission for Asia and the Pacific, 2017[39]). Finally, in October 2020 the World Bank forecast significant remittance falls of up to 14\% following COVID19 (World Bank, 2020[65]). This is potentially significant; in the medium-to-longer term when the economy begins to recover, individuals may increasingly look for solutions like blockchain, which have a competitive advantage in providing cheaper ways to send remittance. 
Nevertheless, important drawbacks remain. For instance, there can be significant 'edge costs' with cryptocurrencies ${ }^{5}$, as transacting into and out of bitcoin to send a payment effectively adds a third currency, and therefore a second foreign exchange swap into each transaction. Linked to this, cryptocurrencies are, as yet, relatively illiquid in emerging economies due to their economic and institutional immaturity. Crucially, the necessity of a computer or smartphone for use can also serve to exclude or even entrench existing inequalities for those who are technologically illiterate or simply lack access to such devices, a point which cannot be overstated. As aforementioned, it is critical that donor government-funded initiatives or investments do not unintentionally widen the digital divide.

Rather, donors should work to (i) inform stakeholders whether there is an appropriate role for blockchain solutions in terms of financial inclusion and/or financial services in the specific given context and (ii) encourage sandbox initiatives (Investopedia, 2020[66]) in which blockchain platforms can work with beneficiary governments to help develop regulation in tandem, allow authorities to vet the platforms and assist in responsible roll-out.

This will avert further incidents such as that of safari.com, which suspended last-mile service to Bitcoinenabled remittances providers on the basis of regulatory concerns and warnings from the Central Bank of Kenya (Parulwa, 2017[67]).

Supply chains constitute a fundamental element of sustainable development, by both linking SMEs in developing countries (formal SMEs constitute up to $40 \%$ of GDP in these contexts (World Bank, 2020[68])) to global markets and facilitating aid disbursement. This relates to both SDG 8 and 9. In particular, SDG Goal 9.3 calls for "increased access of small scale industrial and other enterprises, in particular in developing countries, to financial services, including affordable credit and their integration into value chains and markets" (United Nations, 2015[46]). The COVID-19 crisis exposed the vulnerabilities of existing global supply chains, underlining the importance of better and more effective transparency and traceability. Blockchain-powered solutions can assist donors and their development partners to contribute to SDGs that support economic development whilst undertaking effective due diligence, in line with commitments to uphold Responsible Business Conduct (OECD, 2018[69])

A number of important development-related blockchain pilot in this area already exist. For instance, the World Economic Forum reports that both Asian Development Bank and the Inter-American Development Bank are experimenting with the use of blockchain for single-window projects in South Asia and Latin America, respectively (World Economic Forum, 2020[70]). Elsewhere, AB InBev is employing the BanQu platform in Zambia and Uganda to bring transparency and ensure that local farmers, sometimes referred to as the 'last mile of global supply chains', are adequately paid for their crops (Slavin, 2019[71]). In 2021 and beyond, the pandemic is likely to turn development investors' attention towards harnessing blockchainpowered supply chains to meet long-standing heath challenges in developing contexts. The World Health Organisation (WHO) estimates that as many as one in ten medical products in low and middle-income countries is either substandard or falsified, and the problem stems principally from poor supply chain management, which "prevents local health clinics from knowing the provenance of their medical supplies" (Pisa, 2018[5]). For instance, the Indian organisation Sta-Wig has already entered a pilot to explore the use of blockchain for tracking vaccine delivery in parts of the country (World Economic Forum, 2020[70]).

Although COVID-19 may well accelerate the use of blockchain-powered supply chains, the process of true widespread implementation will likely be more long-term.

This is in part due to the deceptively simple serialisation process, which involves assigning a unique identity to each product in the chain, and is likely to require underpinning international standards on best practice (Pisa, 2018[5]). Added to this, development actors implementing blockchain-powered supply

\footnotetext{
${ }^{5}$ Edge costs occur when 1) the recipient cannot access the digital currency in question directly, 2) there are national restrictions or 3) limited/not yet developed swap contracts.
} 
chains will need to ensure compliance with regulation across multiple jurisdictions (Salmon and Myers, $\left.2019_{[35]}\right)$. Lastly, more specific obstacles include interoperability and scalability. Interoperability again will require robust internationally-agreed standards. Finally, ensuring data integrity, especially in sensitive areas like health, is fundamental, and will be explored in more detail in the following section.

Another element of supply chains for sustainable development is process of aid delivery. Some organisations like Human Rights Foundation and the American Red Cross, have been experimenting with cryptocurrencies in various forms for years (World Economic Forum, 2020[70]). However, recently an increasing number of development actors are joining them in an examination of the utility of digital and cryptocurrencies in streamlining aid delivery. Without doubt "blockchain technology offers tangible benefits to these actors without challenging their roles as central authorities (Reinsberg, 2019 [21]) A recent example is that of the International Federation of the Red Cross and Red Crescent Societies (IFRC), which was awarded a grant to conduct a project exploring the benefits and risks of blockchain-powered humanitarian cash transfer programming. The result was the 2018 Blockchain Open Loop Payments Pilot Project in Isolo County, Kenya, which targeted 2000 drought-affected households with cash assistance (International Federation of Red Cross and Red Crescent Societies, 2018[72])

While benefits and challenges surrounding cash disbursement will be elaborated in more detail in the chapter on four specific examples,

the IFRC pilot in Kenya is worth highlighting as it draws attention to a fundamental issue, which is the difficulty of securing 'meaningful consent' in blockchain pilots (Coppi and Fast, 2019[73).

During the project, consent was captured using mobile phones and recorded on the Red Rose platform (a technology provider for cash transfers in the humanitarian sector). However, unlike the WFP, in which refugees did not need to share sensitive data with mobile operators and third parties, the IFRC pilot in Kenya prompted concerns with the validity of informed consent and, more generally, the compatibility of blockchain with data protection laws and regulation (Salmon and Myers, 2019 ${ }_{[35]}$ ). It is important to underline here that aid was contingent on provision of personal information, and a number of beneficiaries are recorded as expressing concern with this (International Federation of Red Cross and Red Crescent Societies, 2018[72]). Development actors thus need to work harder to secure meaningful consent by deploying blockchain alongside robust technical assistance for local communities. Insofar as possible, the provision of aid should not be contingent on use of blockchain.

Altogether, a cursory examination of blockchain pilots linked to economic development points to the technology's ability to potentially play a key role in enabling donors to help contribute towards at least 7 of the 17 SDGs.

In investing in blockchain-based financial products and services, donors can provide the world's two billion strong unbanked population who are currently excluded from current services run by traditional intermediaries, and who risk falling even further behind as a result of exogenous crises like COVID-19, with access to finance. With the more widespread use of blockchain-powered supply chains, the technology also has the capacity to play a role in the creation of a more resilient, sustainable global economy, characterised by improved produce transparency and the connection of local actors and organisations to global markets, as well as the efficient disbursement of aid. However, at present, obstacles such as unintended exclusion and the difficulty of securing meaningful consent remain pervasive. We therefore recommend that donor governments work closely with beneficiary governments and stakeholders were possible, to address these and other issues related to interoperability and data integrity. With this in mind, donors should work to align pilots with the Istanbul Principles for CSO Development Effectiveness, which call for people's empowerment, democratic ownership and participation in development projects (CSO Partnership for Development Effectiveness, 2020[74]), and the Busan Principles, which encourage 'country leadership and ownership in development strategies' (OECD, 2011 [58]) where possible. 


\section{Blockchain for infrastructure and access to services}

Closely linked to several themes discussed under the 'Empowerment' cluster, blockchain presents the opportunity to improve access to a number of SDGs, presented in Figure 2.4. In Improving 'Infrastructure and Access to Service', we examine blockchain's potential to expand access to education and health institutions, alongside initiatives to mitigate the effects of climate change.

\section{Figure 2.4. How blockchain pilots improve infrastructure and services to contribute to the SDGs}

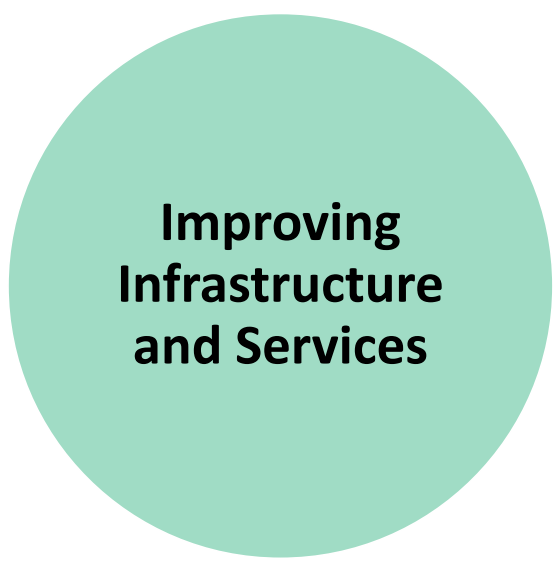

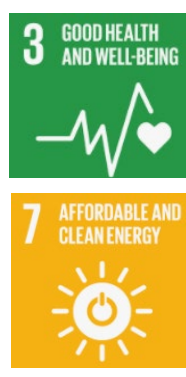
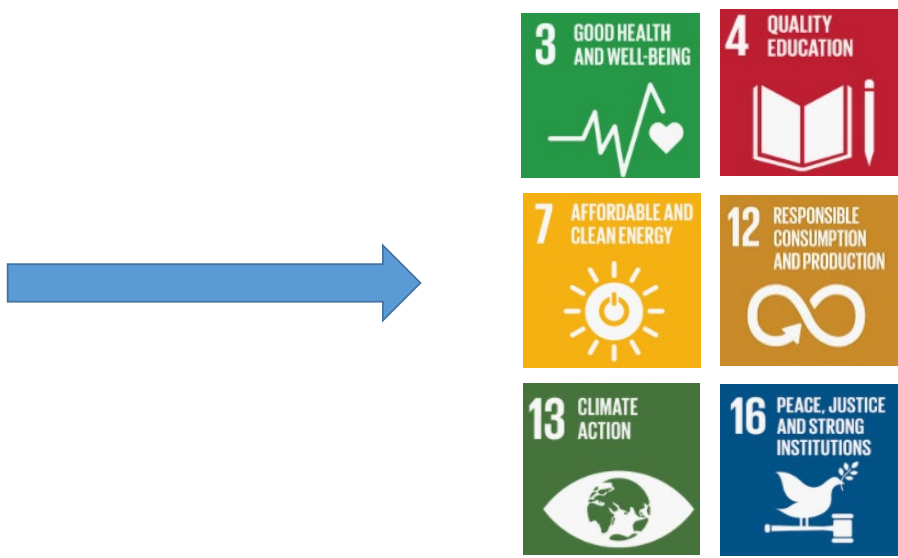

\section{Source: Authors}

For several years, researchers have been investigating to what extent blockchain can implement identity management (including credential and document management) solutions for education and healthcare. On the former, while desk-research of the available literature did not highlight any pilots currently underway, it should be noted that a UK Department of International Development ${ }^{6}$-commissioned report underlined that, blockchain for educational documents is likely to have "strong institutional and technical success" (Mulligan, 2016[9]). In doing so, blockchain can assist donors and development partners in contributing to SDG 4, 'Quality Education' (United Nations, 2015[46]). More specifically, the tamper-resistant nature of blockchain underlines its ability to protect certificates from any potential damage to a country's national infrastructure. The ability to verify educational credentials on the blockchain is particularly significant for certain demographics, such as refugees, who may be fleeing war-torn countries and have lost certifications during the course of their unexpected journeys.

However, the familiar problems of data security and scalability remain a significant obstacle to widespread adoption. For example, educational institutions may require the ability to issue thousands, if not millions, of credentials at any one time during the course of the academic year (GIZ Blockchain Lab, 2019[7]). Linked to this, a fundamental point to note is that virtual immutability underlines the need for accurate data input.

\footnotetext{
${ }^{6}$ As of 2020 , the Department of International Development merged with the Foreign and Commonwealth Office, and is now known as the Foreign, Commonwealth and Development Office (FCDO)
} 
In other words, as long as we rely on human data-entry, there is still the possibility to falsify records by inputting inaccurate data onto the blockchain. Until this issue is resolved by digitised automation, we are unlikely to realise the true revolutionary potential of blockchain, which involves the dis-intermediation of powerful central authorities (GIZ Blockchain Lab, 2019 $\left.{ }_{[7]}\right)$. A separate, individual-level, concern is that if public networks are used, anyone could in theory have access to potentially sensitive information. As such, donors interested in pursuing blockchain pilots for education should experiment with different levels of permission in the design of the base-protocol to minimise this risk.

Like education, blockchain technology also has potentially profound implications for healthcare data management. The data security blockchain provides is critical, especially in a context where errors can represent the margin between life and death (OECD, 2018[22]). The broader benefits of using blockchain solutions for healthcare include the ability to share information easily and securely between different stakeholders. In this sense, healthcare data operations running on blockchain allow greater scope to empower individuals to manage their own files, including sharing with new providers as they choose. Specific development advantages for donors include the ability to contribute to SDG 3 'Good Health and Wellbeing' (United Nations, 2019 ${ }_{[64]}$ ). Further to this, where implemented in state-sponsored healthcare structures, such pilots can help mitigate poor governance models plagued by high levels of bureaucracy and inefficiencies, and therefore help contribute to SDG 16, 'Peace Justice and Strong Institutions' (United Nations, 2015[46]).

For several years now, multiple initiatives for healthcare seeking to provide more robust patient healthcare information data management have started to become more widespread. At present, these tend to be predominantly in developed countries. For instance, Estonia is a notable frontrunner, having integrated blockchain technology in its national health infrastructure with an Electronic Health Record. Elsewhere, Sweden is in the process of developing a national blockchain for health records to give citizens more control over their data (European Blockchain Observatory and Forum, 2018[75]). On the other hand, there is scope for greater investment in blockchain for health data management in developing countries. For instance, across Africa, 26 countries are already known to have eHealth Strategies (Karuhanga, 2018[76]). Added to this, the Stellar (XLM)-based blockchain network (to be used in the creation of Akon's cryptocurrency-based city) has recently partnered with healthcare solution provider Jeev, in order to improve Rwandan's access to healthcare (Crypto Gibraltar, 2020[77]). Following COVID-19's exposure of the fragile nature of many countries' healthcare systems, particularly those in developing countries, we may well see a significant rise of similar projects in the years to come.

Nevertheless, there are significant risks entailed with using blockchain for healthcare data storage. As the OECD Primer states, "while some healthcare blockchain solutions will make only high-level demographic information publicly viewable, it is conceivable that the combination of demographic data and geographic location could reveal sensitive information" (OECD, 2018[22]). Added to this, "given that the information stored (or linked to the blockchain) is highly sensitive, data security is a potential risk". (OECD, 2018[22]) However, on the latter, there are several initiatives working to address some of these concerns. For example, the EU's MyHealthMyData, funded under the EU horizon 2020 programme, aims to use blockchain to create the world's first open biomedical informational network (European Blockchain Observatory and Forum, $\left.2018_{[75]}\right)$. The authors of the EU report in which this is cited emphasise that

Blockchain can serve as a storage full of proofs that off-chain data is in fact genuine.

Now turning to climate mitigation, the vast majority of the current challenges we face in terms of climate and the management of natural resources derive from complex international co-operation problems (Reinsberg, 2020[33]). This includes a fundamental lack of trust and confidence amongst states regarding in the governance and exchange of potential energy supplies (Denis Le Sève, Mason and Nassiry, 2018[30] . Blockchain's potential to solve such fundamental issues could help us revolutionise current models and enable both Donors and their development partners contribute to SDG 7 'Affordable and Clean Energy'; SDG 12, 'Responsible Consumption and Production'; and SDG 13 on Climate Action (United 
Nations, $\left.2015_{[46]}\right)$. In using blockchain for cleaner, greener energy, Donors can also 'build forward greener' post COVID-19, fulfilling their commitments under the Paris Agreement (United Nations Framework Convention on Climate Change, 2016[31] )and the UN Global Compact Principle 8, which calls on donors to "undertake initiatives to promote greater environmental responsibility" (United Nations Global Compact, $\left.2004_{[32]}\right)$.

Today, there are a growing number of blockchain-based initiatives dedicated to climate causes. For instance, the Pacific Alliance is piloting a shared ledger on the basis that it is more cost effective and, through the broadcasting of time-stamped blocks, helps avoid the phenomenon of "double-counting". This, in turn, enables countries to achieve their Nationally Determined Contributions under the Paris Agreement (Reinsberg, 2020[33]). At a more localised level, blockchain can assist individual households in the transition away from centralised, fossil-fuel systems, in favour of peer-to-peer systems powered by renewable energy. One such example in a developed country context is the Brooklyn Micro-grid. Established in 2016 and located in New York City, this project aims to build a peer-to-peer energy exchange in which citizens trade their self-produced energy with each other (Power Technology, 2017 ${ }_{[78]}$ ). There is also increasing evidence in support of micro-grids for energy in poorer communities in developing contexts, like subSaharan Africa and Asia. For instance, the results of a 2018 report indicated the Middle East and Africa would, in the future, be home to the world's fastest market for micro-grids, predicting a total of almost 1145 in existence by 2027 (Energy Storage News, 2020 ${ }_{[79]}$ ). Solshare is one example already in existence, and employs blockchain to power a peer-to-peer energy trading platform in order to bring affordable solar energy to communities in Bangladesh (solshare, 2020[80]).

The blockchain pilots for climate-related SDGs underline the fact that blockchain is not a standalone tool, but rather exists in an ecosystem of inter-related technologies.

For instance, blockchain-powered peer-to-peer energy markets require the use of smart contracts, which "facilitate, validate and record transactions and agreements between multiple parties" (Reinsberg, $2020_{[33]}$ ), and allow contracts to be self-executing when certain conditions have been fulfilled. For energy markets like these, an oracle is necessary to 'feed' the information, in this instance smart metres, to collect process and transmit data. This presents a potential problem in that it ultimately points to the critical need for (i) investment in physical infrastructure that might not already exist in developing country contexts, as well as (ii) resources for monitoring (Denis Le Sève, Mason and Nassiry, 2018[30]).

While the primer highlights, "blockchain-based solution can provide a lower entry barriers and incentivise investors to create more and better renewable electricity generation by providing a highly automated and secure way to buy and sell electricity" (GIZ Blockchain Lab, 2019 $[7]$ ) profound challenges remain. One such example for development actors to overcome is that while public, permissionless blockchain networks are preferable in this instance for their ability to engender higher levels of trust, transparency, and, by extension, co-operation, the energy involved in the consensus mechanisms used in public blockchains is huge. The result could even be a potential net positive energy contribution effect. This risk is much higher in larger networks, which is a further barrier to scale. Altogether, we come back to the issue of baseprotocol design, where there are careful trade-offs to be made. 


\section{Four examples of blockchain for development}

Following the broad mapping exercise conducted in the previous chapter, this section examines four representative examples in more systematic and granular detail. Recognising the surplus of blockchain for development mappings in existence, this chapter aims to dive deeper into concrete examples in order to distil recommendations for policymakers. Such recommendations are more important than ever as COVID-19 accelerates the transition to a 'digital by default' society. Analysis is based primarily on desk-research, and complimented by in-depth interviews with individuals directly involved in the projects. Each example discusses (i) the development context, (ii) the blockchain solution and why it was chosen, (iii) implementation challenges and criticism, (iv) advantages for donors and development partners and (v) potential future applications.

Readers should note that the selected case studies found below are illustrative only, and inclusion does not represent OECD endorsement. 


\section{Building blocks - a World Food Programme (WFP) blockchain system for cash transfers}

\section{The development context}

Traditionally, humanitarian assistance has sought to mitigate some of the worst effects of crises by distributing relief materials. However, following the World Humanitarian Summit in 2016, which emphasised the importance of localisation and innovation (Devex, 2020[81]), cash-based approaches have become increasingly common for the provision of humanitarian assistance. Cash-based approaches allow rapid response at scale, directly providing beneficiaries with the financial means to enable them to buy relief materials (Office of the United Nations High Commissioner for Refugees, 2020[82]).

By permitting more freedom in the use of resources, cash-based approaches enable beneficiaries to fulfil their needs in a more independent and dignified manner, (Office of the United Nations High Commissioner for Refugees, 2020[82]), thus putting the 'human' element back into humanitarian assistance.

Research also indicates that cash-based assistance is more cost-effective, with lower cost per beneficiary than in-kind transfers, and has a positive impact on the local economy (Doocy and Tappis, 2017 [83]; Office of the United Nations High Commissioner for Refugees, 2020[82]). A study comparing voucher programmes and cash transfers demonstrated that whilst voucher programmes generated up to USD 1.50 of indirect market benefits for each USD 1 equivalent provided to beneficiaries, cash transfer programmes generated more than USD 2 of indirect market benefits for each USD 1 provided to beneficiaries (Doocy and Tappis, $2017_{[83]}$ ). More broadly, cash transfers support the recovery of livelihoods by providing purchasing power and thus re-stimulating local demand. Altogether, this has a positive economic multiplier effect when implemented at scale (Creti and Jaspars, 2006 ${ }_{[84]}$ ). Cash transfers also enable beneficiaries to cover the most important household needs and improve household food security and nutrition (CARE, 2017[85]; Harvey and Pavanello, 2018[86]).

Cash based humanitarian assistance often takes the form of cash transfers and thus requires the intervention of a third party, such as a financial intermediary. These can include banks, mobile money companies, and local agents. The process of transferring money to millions of beneficiary accounts through a financial intermediary is costly and inefficient (Lucsok, 2018[87]) (Zambrano, Young and Verhulst, 2018[88]).

In a normal cash transfer setting, WFP would provide information (refugees) to the local financial intermediary (often a bank), who then opens and manages the beneficiaries' bank accounts. WFP would then the bank upfront with the total cash (to fill the bank accounts) needed to purchase food. The bank subsequently informs the refugees that a bank account has been opened in their name, and provides them with a debit card or e-voucher. The refugees are thus able to go to a local store and purchase food. The bank validates the transactions, settles with the retailers at the end of every month and provides a summary of accounts to WFP.

Overall, the entire process of working with local and regional banks can be costly and inefficient (Lucsok, 2018[87]; Zambrano, Young and Verhulst, 2018[88] $)$. In addition to associated costs, the underdeveloped financial systems in some countries and unstable operations of banks renders the transfer processes even more risky and uncertain. As there is a need to create a bank account for every beneficiary, there are privacy concerns and risks associated with sharing confidential information of refugees with the local financial institutions (Smith, 2019[89]).

\section{The blockchain solution}

The World Food Programme (WFP) was the first leading humanitarian organisation to use a blockchainbased cash transfer system, with the aim of making humanitarian assistance more efficient, effective and 
secure. The implementation of innovative technologies like blockchain for food assistance exemplifies the ability of development actors to leverage technology to advance progress towards the Sustainable Development Goals (SDGs). In the case of WFP, their blockchain project targeted SDG 2, 'Zero Hunger' (United Nations, 2015[46]). WFP began their blockchain initiative, 'Building Blocks', in 2017 with 100 people in Pakistan. Following promising initial results, WFP expanded the scale of the project, delivering money to 10000 Syrian refugees in Jordan. This was later expanded to 160000 refugees. Now covering almost all the Syrian refugees in Jordan, and 300000 refugees in Bangladesh, Building Blocks is undoubtedly the largest and most sophisticated implementation of blockchain technology for humanitarian aid.

Building blocks, which is built on a private, permissioned blockchain, was developed in order to ensure necessary security and privacy for refugees benefiting from the programme (World Food Programme, 2020[00]).

WFP's Building Blocks uses blockchain technology as the back-end infrastructure to create virtual accounts for beneficiaries on the blockchain and upload their respective entitlements. Once a virtual account has been created, a beneficiary is able to go to a registered supermarket, and purchase goods by performing an iris scan (in the case of Jordan project) to verify and complete the transaction. This process is deceptively simple; the back-end is built on a complex web of blockchain technology.

To clarify, in the back-end, blockchain performs the role of a financial institution. It authenticates ${ }^{7}$ the individual's identity via a linked relational database with hashed information about beneficiaries and their entitlements (Zambrano, Young and Verhulst, 2018[88]). This verification helps to ensure that the individual purchasing goods is actually entitled to access the resources. Upon the verification of identity, the transactions are performed and subsequently recorded on the blockchain ledger. In practice, this means that the amount of purchase is automatically sent to the blockchain ledger, which authenticates the transaction and sends a confirmation back to the supermarket to issue the receipt (Kenna, 2017[91]). The blockchain ledger records the amount owed to each refugee by WFP and, after each purchase, records the amount owed to the vendor by WFP (Smith, 2019[89]). Based on this transaction history, WFP then settles outstanding balances with the vendor every month.

The entire accounting, validation and reconciliation takes place on the blockchain, and the final settlement is through normal bank transfers to the vendors. As a result, the WFP no longer needs to transfer upfront the whole amount of the cash transfers programme to the local financial institutions. Instead of transferring money to a bank and then from bank to thousands of accounts, the information is recorded on blockchain and WFP simply makes 200 bulk payments to the registered vendors every month.

\section{Implementation challenges and criticism}

In avoiding the use of cryptocurrencies or stablecoins ${ }^{8}$, WFP largely avoided regulatory and judicial challenges. WFP stated they decided to not use cryptocurrency due to their volatility, illegality in certain jurisdictions, and lack of adoption.

Arguably, the most fundamental challenge the initiators of the scheme faced was reluctance to change existing models. Reluctance to alter a well-established process and potentially run the risk of curtailing

\footnotetext{
${ }^{7}$ Although WFP utilised iris scans as the verification process in Jordan, the authentication method is flexible. In Bangladesh, for example, WFP used fingerprints, shifting to QR codes following the emergence of the COVID-19 pandemic in order to comply with social distancing measures. More broadly, the authentication method is dependent on the accuracy level needed by the donors. Biometric scan is the preferred method if donors prefer a fool proof system to reach the intended beneficiaries. However, in comparison to mobile phone or a one-time password verification, the implementation of biometric scan system is relatively expensive. Amidst the pandemic, in order to comply with social distancing measures and minimise the time spent by refugees in public places, WFP introduced a commodity voucher based on a fixed basket of goods based on calculated nutritional value of commodities.
}

${ }^{8}$ Stablecoins are a form of cryptocurrency that peg their value to an external reference 
refugee agency by limiting the method of cash access to a digital solution based on a frontier technology. Consequently, programme designers were tasked with convincing both internal colleagues and external donors, influenced by the mainstream misperceptions surrounding blockchain, that the scheme was viable. Navigating this space, WFP followed an incremental and results-oriented approach in order to convince donors and attract funding to expand the scale of their operations.

While the first Building Blocks pilot in Pakistan was built on public Ethereum blockchain, the project architects quickly found that transactions on Ethereum were too slow and incurred disproportionately high fees. The subsequent switch to the current private permissioned blockchain, based on proof-of-authority consensus mechanism has enabled faster and cheaper processing of transactions. WFP can see and track the transactions taking place in real time, whilst controlling who joins the network. The process is also centralised (atypical for a decentralised blockchain). Although WFP has limited the role of banks in development aid, in certain respects it has essentially adopted this function itself (Juskalian, 2018[92]). Altogether, critics assert WFP's model is more of a traditional database than a real shared blockchain solution, given that it is a private, permissioned blockchain, which rests on proof of authority (Juskalian, 2018[92] $)$ However, the specificities of the protocol design were most appropriate for the situation; they followed careful consideration of the end-beneficiaries and their needs, as well as the programme's overall development impact objectives.

Until there is internationally wide spread adoption of blockchain, tying refugees to digital finance potentially risks excluding them from traditional financial services, or at least creating a significant barrier. In the most extreme scenario, pivoting away from the traditional financial sector may even undermine the cause of financial inclusion as it was conceived in the SDGs. Other, more recent, research has suggested that even though the WFP model employs a private, permissioned blockchain, the hashed information about the beneficiaries and their entitlements still presents a potential data infringement risk (Coppi and Fast, $2019_{[73]}$. The organisation is still effectively managing sensitive information on behalf of individuals, which, if a data breach did occur, could lead to further beneficiary persecution and discrimination (Christie, $\left.2020_{[27]}\right)$.

\section{Advantages for donors and development partners}

Overall, WFP's Building Blocks demonstrates how donors can employ blockchain to accelerate the SDGs. For instance, the programme directly targeted SDG 3 'No Hunger'. Nevertheless, more broadly, institutional implementers assert that it supported the local economy, thus indirectly targeting SDG 8 'Decent Work and Economic Growth'. Further to this, in empowering refugees to assert their self-sovereign identity, Building Blocks arguably contributes to SDG Goal 16.9, under 'Peace, Justice and Strong Institutions', which calls on countries to, "by 2030, provide legal identity for all". Finally, the involvement of refugees as the beneficiaries ensured the programme upheld the underlying 2030 Agenda commitment of 'leaving no one behind' (United Nations, 2015[46])

While the multiplier effect of the Building Blocks programme does not necessarily outweigh that of traditional cash disbursement writ large, arguably, the blockchain-based solution's true advantages lie in more practical considerations.

For example, the use of blockchain technology as part of the aid disbursement process renders the traditional role of banks redundant, thus effectively eliminating transactions costs. The pilot project in Jordan eliminated a staggering $98 \%$ of the bank-related fees, equating to USD 4000 a month for the World Food Programme (World Food Programme, 2017[93]).

Linked to this, there is no need to pay local financial intermediaries for the creation of beneficiary accounts, as well as services such as reconciliation and monthly reports (Kenna, 2017[91]).

Another advantage with the use of blockchain technology is the reduction of privacy-related risks associated with sharing personal refugee details with the local financial institutions for the creation of 
accounts. The traditional method of working through financial institutions is also time consuming, particularly with regards to on-boarding beneficiaries. However, with this new system in place, the creation of virtual accounts is easy and efficient.

It is worth noting that in the future, WFP plans to expand and include other organisations in the programme. Some actors have raised concerns about potential monopoly, accessibility to such a partnership and question the willingness of organisations' to allow WFP to effectively host other organisations data, are still to be finalised. However, as the current aid delivery space is severely fragmented, characterised by a multiplicity of donors and actors with sometimes competing goals and methods (Klingebiel, Mahn and Negre, 2016[94]), such an approach would help minimise competition between development actors, establishing Building Blocks as a more neutral ecosystem, promoting greater collaboration and peer learning. This would also follow recent trends within the international development community towards greater collaboration and transparency around funding, as demonstrated by the Development Cooperation Profiles (OECD, 2020[95]) and the International Aid Transparency Initiative (International Aid Transparency Initiative, 2020[96]).

\section{Potential future applications}

Beyond Jordan, WFP also employs blockchain technology in its work in Bangladesh, which currently covers 200000 refugees. As of summer 2020, there were plans to scale this number and eventually cover 850000 refugees in Bangladesh. ${ }^{9}$ It is worth emphasising that COVID-19 is set to accelerate the less than $20 \%$ of humanitarian relief currently delivered through cash-programming, as it represented the most commonly used method of immediate social assistance in the wake of COVID-19 (Devex, 2020[81]). As such, blockchain-based programmes like Building Blocks, with clear stated aims around improving the efficiency of development, are well placed to assist the acceleration of cash-programming.

Building Blocks itself plans to scale up and establish a system where every beneficiary has a digital identity with complete ownership and control over their own data. WFP envisions a world where every beneficiary can use this digital identity to access services, enter economy and live a dignified life. It is hoped this blockchain-based solution piloted in a cash-disbursement context will be used for a whole host of applications, including but not limited to, recording education and employment credentials, the creation of credit profile, and recording land titles. All these applications can be used by the beneficiary to start a new life in a new country. Even without documents, with this system in place a refugee would not have to start the process again from scratch in a new country (Juskalian, 2018[92]).

\section{Unblocked cash: cash disbursement in Vanuatu}

\section{The development context}

The Pacific falls behind other regions in the use of cash transfers for humanitarian assistance, primarily due to poor financial services infrastructure, lack of local capacities and lack of access to global training related to accounting and management of cash transfer programmes. Debit cards are not widely used or accessible in the Pacific, and online financial services are even rarer.

In addition, implementing cash and voucher based assistance (CVA) is complicated, especially in developing country contexts. This is due to the lack of financial services and payment instruments, rural and marginalised populations without photo identification, as well as generalised low rates of digital and financial inclusion.

\footnotetext{
${ }^{9}$ Information provided by WFP
} 
More generally, implementing CVA presents issues related to the high costs linked to monitoring and reconciliation, payment processing and recipient registration (Rust, 2019[97]), as already presented in the preceding WFP case. These costs are even higher in the Pacific context, particularly Vanuatu, where the cost of cross-border and bank-to-bank transfers is extremely high. To use remittance services as an example, rates to send and receive money remain amongst the highest globally. As many pacific island countries are multi-island environments, the associated costs of monitoring are also higher than in what we might refer to as mainland countries.

\section{The blockchain solution}

'Unblocked Cash' is a blockchain-based pilot set up by Oxfam ${ }^{10}$, Sempo ${ }^{11}$ and ConsenSys ${ }^{12}$ to make CVA more efficient in Vanuatu, and to enable a more rapid scaling of CVA across the Pacific region in a manner that is less costly and more automated, enabling uptake by smaller development partners and country teams. An initial pilot was conducted for 1200 households' mid-2019, and is now being expanded and scaled to serve as the primary platform to deliver CVA for a COVID-19 and tropical cyclone response, covering nearly 35000 beneficiaries and over 500 vendors across 15 islands. Recently, the project was awarded the EU Horizon 2020 Prize for Blockchain for Social Good in the Aid \& Philanthropy Sector, providing EUR 1 million for future scaling.

The blockchain based solution used by Unblocked Cash is broadly similar to the one deployed in the WFP example presented above. However, Unblocked Cash adds three critical elements: the use of stable coins, the deployment of smart contracts, as well as its status as a multi-stakeholder, multi-country CVA delivery platform.

Unblocked Cash works as follows (ConsenSys, 2019 [98]; Rust, 2019 $9_{[97]}$ ). Each beneficiary receives a Prefunded 'Near Field Communication (NFC) card'13 with the tokenised value for goods and services in the

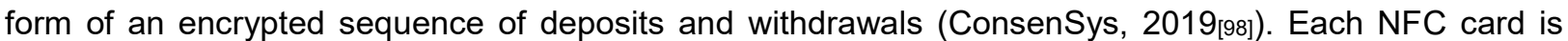
mapped one-to-one with an address on the Ethereum main net, where the beneficiary's balance is held (Rust, 2019[97]).

To be on-boarded, vendors must provide identification documents, bank account details and a photograph, fulfilling Know Your Customer (KYC) requirements, in line with Vanuatu's financial regulations. Upon the completion of the registration process, vendors are given smartphones (NFC compatible devices) with the Sempo app that allows to process contactless payments (ConsenSys, $2019_{[98]}$ ). The beneficiaries can then go and buy goods on an unrestricted basis from registered vendors, using the NFC card, and thus their 'tokenised' cash. Following an agreement between the vendors and Oxfam, the latter repays the vendors in their local currency, effectively 'off-ramping' the stablecoin from the platform and enabling an equivalent conversion into local currency.

To avoid the high transaction costs associated with cashing out a large number of small vendors, ${ }^{14}$ Oxfam also introduced the concept of 'super vendor', a village shopkeeper who holds enough cash funds to cash

\footnotetext{
10 Oxfam is a confederation of 20 independent charitable organisations focusing on the alleviation of global poverty (Oxfam, 2020[112]).

11 Sempo is an Australian-based start-up with a mission to efficiently distribute relief funds to at-risk communities across the globe while increasing financial inclusion (Sempo, 2020[113]).

12 ConsenSys is "global blockchain technology company building the infrastructure, applications, and practices that enable a decentralized world" (ConsenSys, 2020[114]).

13 Near-field Communication (NFC) is a standardised contactless communication technology based on a radio frequency (RF) field (Rust, 2019[97]).

${ }^{14}$ Each bank transfer costs AUD 20.
} 
out multiple smaller vendors. In a subsequent iteration of the programme for COVID-19 response, Oxfam expanded this function to enable all enrolled vendors to pay or cash out other vendors using the same arrangement, thereby reducing the liquidity burden on a single vendor.

The Sempo dashboard used to monitor the disbursements in the backend also verifies the transaction before recording them on the ledger. The one-to-one mapping of the NFC to the Ethereum enables Sempo to submit the transactions to the ledger. A smart contract is used to verify that the single Ethereum account controlled by Sempo is the only author of transactions in the ledger, and that transactions can only be completed by whitelisted vendors and registered beneficiaries fulfilling minimum requirements (registration data).

The Unblocked Cash project uses DAI stablecoin to ease the reconciliation process. ${ }^{15}$ This implies that upstream, Oxfam converted all of its relevant budget line allocated for the cash transfer amount into a cryptocurrency. This system presents three main advantages.

First, DAl stablecoins are protected by what is called a 'crypto collateralised voucher' (CCV).

This complex system in practice protects the cryptocurrency, so that only the participants within the Sempo system who have had KYC checks can use it. This allows the DAI to comply with anti-money laundering regulation, and also automates the verification, authentication and reconciliation processes associated with the use of vouchers in CVA, in a manner that streamlines these processes, thus reducing complexity and cost.

Second, using the DAI allows to avoid risks associated with the volatility of the local currency, particularly in small and relatively undiversified economies, prone to inflation and currency fluctuation.

This also effectively protects participating beneficiaries and vendors from losing purchasing power and revenue due to these fluctuations.

Lastly, the use of smart contracting to enable electronic vouchering also addresses the cost inefficiencies linked to lengthy and complex reconciliations that have hindered voucher-based programming in the humanitarian sector. The issuance of an e-voucher (as opposed to a payment) to beneficiaries requires fewer KYC identification checks, making the system more accessible to those without formal ID, and reducing NGO costs incurred conducting identification checks, as these are only applicable to vendors. The e-voucher also increases security by eliminating the need to carry cash, while also directing beneficiary expenditure to small local vendors in a highly traceable manner.

\section{Implementation challenges and criticism}

In the initial stage, the project faced significant regulatory challenges, as Vanuatu had placed a moratorium on the use of blockchain technology and cryptocurrency due to a number of cryptocurrency scams. In parallel, the team also experienced resistance within Oxfam, as blockchain technology was perceived as highly risky.

In response, the team worked with the Australian-based EY blockchain team to analyse, assess and, ultimately, establish compliance procedure appropriate both within and outside Vanuatu.

As a result, it was possible to get the approval to implement the project from the Reserve Bank to implement the project for humanitarian purposes. Based on the success of the pilot, the government's Distributed Ledger Technology Task Force amended the moratorium on the use of blockchain technology and cryptocurrency.

\footnotetext{
${ }^{15}$ A stablecoin is a form of cryptocurrency that is pegged on a 1-1 basis with a global reserve currency. In the Unblocked Cash case, DAI is pegged to the US dollar. This avoid the volatility associated with some cryptocurrencies, such as Bitcoin, and ensures that funds maintain a value associated with a stable global currency.
} 


\section{Advantages for donors and development partners}

There are a number of advantages for donors in using this system. First, the use of Unblocked Cash has resulted in cost reductions for cash transfers in Vanuatu of over $50 \%$. In addition,

the system provides donors with high-quality, timely and accurate reporting of how their resources were used. The advantage for donors of using this system is significant, as reporting is directly linked to aid accountability.

Unblocked Cash has resulted not only in cost savings, but also in improved transparency in the use of the funds (ConsenSys, 2019[98]). Overall, the pilot is well aligned with several of the Grand Bargain commitments required of Donor countries and, as it was designed with the 'Commitments for Cash Transfer Programming' proposed by Cash Learning Partnership (CaLP) in mind. The system includes access to a central dashboard where transactions can be tracked using automated analytics, including filters for purchase categories, gender, targeting criteria, location and time period. Access to the dashboard is permissioned, enabling limitations on who can issue e-vouchers and 'off-ramp', or withdraw, vendor funds for payment; this ensures data protections and fraud mitigation measures are in place. Donors are also given the option to access the central dashboard using a 'view only' function, which anonymises beneficiary data but enables real-time visibility.

\section{Potential future applications}

The pilot project in Vanuatu distributed VUV 966 443/AUD 11896 to 187 households (1 200 people) and 27 vendors in 2019. In 2020, Oxfam scaled the initiative within Vanuatu and across multiple country programmes.

Oxfam is fulfilling plans to scale the project across the global confederation, and is currently running pilots in Papua New Guinea and the Bolivarian Republic of Venezuela to continue to iterate and improve the approach, as well as enable speedier CVA delivery in contexts where CVA has previously been problematic. Likewise, an additional pilot funded by the Australian government is planned in the Solomon Islands for the first quarter of 2021.

Finally, with the EU Horizon 2020 Prize, Oxfam in the Pacific and Oxfam Ireland have partnered to build internal capacity to support continued scale, including a global Unblocked Cash team and a Pacific Education, Innovation and Learning Hub to support continuity and share experience.

\section{TruBudget - a KfW initiative}

\section{The development context}

The use of partner country systems for aid monitoring and reporting can present multiple problems for donors. First, donor countries often perceive a very high risk of misuse and misappropriation of donor funds in the partner countries (Mckechnie and Davies, 2013[99]). There is a risk that partner countries could be diverting aid, and thus hindering the achievement of development results. For example, Scandals like 'Malawi cash gate' - where it was estimated that about USD 32 million was lost to fraudulent payment through a technical loophole in the public financial management system - have only served to increase the concerns of donors. More recently, of the 102 countries that have received funding for the Global Fund to fight COVID-19, several stand accused of misappropriation of funds (Aidspan, 2020[100]). In Kenya, for example, these claims centre on the Kenya Medical Supplies Authority, who allegedly flouted procurement regulation (Aidspan, 2020[100]). Second,

Occasionally partner countries' public financial management (PFM) systems lack the levels of transparency and efficiency required by donors. 
Finally, some donors are preoccupied with the reputational risk of localising aid. In other words, donors fear that damage to their image and credibility if partner countries manage the funding provided independently, but do not successfully meet the development objectives the donor is accountable for delivering (OECD, 2010[101]).

Consequently, donor institutions have established parallel structures with own rules and procedures for aid monitoring. This results in a large proportion of time-consuming administrative processes, as well as high transaction costs concerning donor co-ordination and management of funds (KfW, 2019[102]).

The multiplicity of aid donors and the different institutional channels through which aid flows also generates direct and indirect transaction costs for the partner countries. The division of aid into small fragments, with each of strand requiring separate negotiation, management, and reporting requirements as well as the complexity of maintaining relationships with multiple donors, adjusting to different procedural requirements, requires significant time and resources (Acharya, de Lima and Moore, 2006 ${ }_{[103]}$ ). Sometimes, the partner country treasury might not even know the status of a disbursement or what money is coming in (OECD, $\left.2010_{[101]}\right)$, jeopardising the process of national investment budget planning.

Donors' failure to leverage local partnerships also undermines commitments made in the Busan Partnership for Effective Development Co-operation, which call for the use of country systems for monitoring and reporting aid, and calls for an improvement in partner countries' PFM systems (OECD, 2011 [58]).

\section{The blockchain solution}

KfW's Trusted Budget Expenditure Regime (TruBudget) software aims to increase the use of country systems for aid monitoring and reporting by addressing the low levels of transparency in partner countries' PFM systems, one of the two main issues raised by donors.

TruBudget is a "blockchain-based digital workflow tool for partner countries, which allows for transparent and efficient collaborative project implementation" (KfW, 2019 $9_{[102])}$.

The TruBudget platform allows to track each action and disbursement made by each stakeholder involved in a project or programme. The result is that, for example, each donor can see how the partner country is deploying the aid resources to contribute towards agreed development objectives.

User-friendly and is easy to use with an interface, TruBudget was conceived as a complimentary tool and may be integrated into the individual IT systems of stakeholders via APIs. This links it to the existing systems and internet connection, which does not have to be fast or regular. Often, the countries trying to use this system are concerned about changing their IT systems, but TruBudget interface enables connection with existing management and accounting tools.

\section{Implementation challenges and criticism}

The main difficulty in implementing TruBudget has been encouraging uptake. For instance, the donors need to be convinced that the advantages in giving partner countries greater independence when using their country systems and structures outweigh any potential risks. In this sense, donors are tested on how much they are willing to support and promote the structural independence of developing countries (Kleffmann, 2019 $\left.{ }_{[104]}\right)$. The partner countries, are incentivised to improve their reputation both in terms of the international community and their own populations, by demonstrating their trustworthiness. By fostering trust and accountability, the use of TruBudget has the potential to simplify and harmonise procedures and modalities in the long run.

However, the resistance to change is a big challenge and changing procedures requires strong commitment, convincing stakeholders over time. 


\section{Advantages for donors and development partners}

TruBudget improves transparency, thus enhancing trust among partners, in three principal ways. First, through blockchain, each action is recorded in a tamper-resistant fashion.

On this particular blockchain, every user action is stored on a shared ledger, visible to all. Increased transparency also serves to improve the quality of PFM systems, increasing trust and reducing risks, as well as ultimately helping donors to fulfil Busan Partnership commitments (KfW, 2019 [102]).

Second, the use of blockchain technology also ensures that there is no monopoly of control over data. A general problem with centralised databases is that the managing organisation may change the data stored, which undermines trust in the system. However, in this case, trust is derived from the decentralisation and immutability of the blockchain solution. Once entered, information cannot be lost and is protected from retroactive forgery (Kleffmann, 2019 $9_{[104]}$ ). Data shared with third parties is thus reliable and trustworthy.

Third, all activities are documented in the system and are accessible and traceable at all stages. The software allows real-time information to be shared among the users and makes it possible for every partner to access latest information about the status of every project.

TruBudget also reduces the costs of managing projects and programmes. The new system lowers transaction costs associated with cumbersome coordination efforts and reduces email correspondence, like requesting updates and sharing reports (Kleffmann, 2019 $9_{[104]}$ ). This advantage has assumed even greater importance in light of the COVID-19 pandemic, particularly in the context of narrowing fiscal space and attendant pressures on aid budgets.

TruBudget allows donor countries to use partner country systems, enabling donor money to be included directly and safely in a partner country's budget process. This approach is effective, sustainable and strengthens partner country's long-term capacity to develop, implement and account for its own policies (Kleffmann, 2019[104]; OECD, 2010[101]).

TruBudget software code is open and is available to anyone, free of charge.

\section{Potential future applications}

The software could, in theory, also be coupled with other technologies, like artificial intelligence, big data to analyse the trends and measure the impact of the projects (Kleffmann, 2019[104]). So far there are six projects using TruBudget, and each of them are at different stages of implementation: Burkina Faso's Ministry of Finance established its own version called SIGFE (Système Intégré de Gestion des Fonds Extérieurs), with a view to extend it to all donor-funded projects. The Brazilian Development Bank also use TruBudget in their IT systems to manage the Amazon Fund. Similarly, Georgia and Ethiopia are planning to use TruBudget for KfW-financed projects. Likewise, GAVI, The Vaccine Alliance is exploring the use of TruBudget for financial management as well as in the vaccine supply chains (Kleffmann, 2019[104]).

\section{Seso Global - a one-stop property shop}

\section{The development context}

The limited number of countries with functioning, country-wide land administration systems is a huge barrier to sustainable economic development (GIZ Blockchain Lab, 2019 $9_{[7]}$ ). For instance, while Africa comprises roughly $20 \%$ of the world's land-based assets (Bolt, $2019_{[105]}$ ), only $10 \%$ of its surface area is formally documented (GIZ Blockchain Lab, 2019 $9_{[7]}$ ). This problem stems from incomplete and often conflicting legacy systems, which are often hampered by a lack of transparency and access to reliable land market data. 
Signs declaring "This Land is Not for Sale" are a common feature across Africa, particularly in countries like Nigeria, where the government's land ownership records are not deemed sufficiently trustworthy. Occasionally, this can even lead to violent disputes.

These systemic problems serve to keep up to USD 100 billion worth of capital trapped in Nigeria alone (Business Insider by Pulse, $\left.2019_{[106]}\right)$ In nearby Ghana, an overwhelming $80 \%$ of land market activity remains unknown and uncaptured. (Bolt, 2019 ${ }_{[105]}$ ) Where tenure is documented, registration is often completed through centralised, paper-based processes, which are cumbersome and rife with suspected corruption. For example, registration with the Ghana Lands Commission, the country's centralised register, is a long and expensive process which can take up to five years and involve multiple different agencies (Hacibeyoglu, 2008[107])

As well as creating a significant obstacle to sustainable economic development, lack of land security tends to disproportionately affect society's most vulnerable, including women, the poor and marginalised groups such as indigenous people (GIZ Blockchain Lab, 2019 $9_{[7]}$ ). While the World Bank has been collecting information on national land administration systems for its 'Doing Business Indicators' since 2004, it is often difficult for partner governments to manage and find workable solutions for these deeply embedded land tenure issues. The result is a long-term, persistent insecurity that contributes to overall economic instability.

\section{The blockchain solution}

Seso Global (Seso) ${ }^{16}$ is a British company that seeks to address the trust deficit between the governments of Nigeria, Ghana, and South Africa and their respective populations, concerning land tenure with an innovative blockchain-based solution.

Seso Global is a self-described 'one-stop shop property portal', powered by blockchain technology. Seso is an ecosystem of services which aims to create added value for property sectors by linking buyers with a multitude of stakeholders across the real estate value chain, including property developers, mortgage provider, banks, law firms and insurance providers. (Vallee and Yu, 2020[108]) Seso currently works with 70 credible property developers across these countries, and has 7000 units on the platform.

The system works to address specific buyer concerns which emanate from emerging markets marred by a lack of trust. For example,

Seso employs law firms to perform KYC checks on both developers and sellers, architects to ensure the property was built with the correct materials, as well as valuers, insurers and banks to verify that the property is bankable. Altogether, the platform works within the respective countries' legal and regulatory frameworks to build up a solid network base and property sector expertise.

In this context, blockchain acts as a tool to timestamp data and ensure immutability; to store information only after the verification process is complete. In this sense, blockchain aids in the creation of what may eventually become an entirely digitised property registry.

\section{Implementation challenges and criticism}

In relying on off-chain verification, arguably Seso does not necessarily unlock or leverage blockchain's full revolutionary power. However, trusted middlemen will continue to perform checks and verification in land contexts until a whole digital architecture of smart contracts and oracles is established to facilitate automated processes (GIZ Blockchain Lab, 2019 $\left[{ }_{[7]}\right)$.

\footnotetext{
${ }^{16}$ Based in Ghana, Nigeria, South Africa and the United Kingdom, SESO is a Digital Lands Transactions platform that facilitates secure, trusted and risk-free Blockchain Land Transactions between, Property Owners, Land Service Agencies and Financial Institutions: (Seso Global, 2020[115])https://app.seso.global/properties/home
} 
Second, while the lack of established legacy systems in many emerging economies reduces the cultural resistance to the creation of digital land registries, the use of public blockchains for such contexts still involves tangible risks. Perhaps the most important here, is that blockchain is still classified as a frontier technology, and has only been in existence for just over a decade.

Given that land titles can sometimes be kept for decades if not centuries, we need to start thinking long-term about solutions to potentially migrate data and improve interoperability (GIZ Blockchain Lab, 2019[7]).

Third, although blockchain-based digital solutions for land tenure can benefit those traditionally "left behind" by the current system (or lack thereof), it remains largely unexplored as to whether blockchain-based land ownership can be integrated into traditional, or non-western land governance systems (GIZ Blockchain Lab, 2019 $[7])$. Linked to this,

projects with a long-term aim of effectively revolutionizing institutional governance systems must be accompanied by technical assistance, to ensure the local population is sufficiently digitally literate to receive the full benefits of the technology.

At all costs, such projects must avoid unintentionally exacerbating the digital divide by de facto only being available to those with a certain level of technological understanding and awareness.

Finally, any donors considering investing in blockchain-based land tenure solutions should take care to perform due diligence checks on energy consumption, and seek ways to appropriately mitigate this.

\section{Advantages for donors and development partners}

In seeking to bridge the trust deficit in emerging markets, projects like Seso can help donors work towards SDG 16 'Peace Justice and Strong Institutions' (United Nations, 2015[4]]). Securing land registries can also help partner countries to better mobilise resources by leveraging land tax, particularly important in today's financially constrained global context.

Fundamentally, Seso is about broadening financial inclusion.

Real estate (and land transactions more widely) constitutes one of the formal financial services, like banking and remittances, that many individuals in emerging economies struggle to access (Medium, $\left.2017_{[109]}\right)$. The primary reason for this is that institutional barriers stemming from legacy systems render the cost of entry simply too high. Reducing the cost of performing real estate and land transactions allows for greater participation in the market. In this sense, blockchain-based land tenure solutions can help donors and development partners work towards SDG 1 'No Poverty', SDG 5 'Gender Equality' and the overarching Agenda 2030 commitment to 'leave no one behind' (United Nations, 2015[4] $)$.

\section{Potential future applications}

In Nigeria, one of Seso's key markets, the company is developing a mortgage scheme specifically targeted at the country's large diaspora community. Tapping into this demographic opens up a significant investment source, as $50 \%$ of the USD 46 billion in remittances that were sent to sub-Saharan Africa in 2018 flowed to Nigeria. Short-term, a diversion could risk a fall in remittance numbers. However, as the World Bank concluded in the Caribbean case, long-term,

Redirection of diaspora funding into investment has the potential stimulate economic growth and job creation in the region. (World Bank, 2016[110]).

If this logic were to prevail, such a step could help make tangible inroads towards SDG 8 'Decent Work and Economic Growth'. 


\section{References}

Aburass, S. (2017), "Syrian refugees' documentation crisis",

https://www.nrc.no/news/2017/january/syrian-refugees-documentation-crisis/.

Acharya, A., A. de Lima and M. Moore (2006), "Proliferation and fragmentation: Transactions costs and the value of aid", Journal of Development Studies, Vol. 42/1, pp. 1-21, http://dx.doi.org/10.1080/00220380500356225.

Aidspan (2020), Misuse of COVID-19 Funds Unvermines the Fight Against the COVID-19 Pandemic, https://www.aidspan.org/en/c/article/5263.

BanQu (2020), BanQu and Zambian Breweries Empower Local Farmers, https://banqu.co/presscoverage/banqu-and-zambian-breweries-empower-local-farmers/.

BBC (2019), Crypto-currencies gaining popularity in Kenya, https://www.bbc.com/news/business-47307575.

Bitdegree (2018), Proof of Work VS Proof of Stake: Which One Is Better?, https://www.bitdegree.org/crypto/tutorials/proof-of-work-vs-proof-of-stake.

Blockchain News (2020), European Central Bank President: Coronavirus Has Accelerated Digital Currency Adoption, https://blockchain.news/news/european-central-bank-presidentcoronavirus-accelerated-digital-currency-adoption.

BlockXLabs (2018), Blockchain and the Sustainable Development Goals, https://medium.com/hackernoon/blockchain-and-the-sustainable-development-goalsc51c52e0af28.

Bolt, J. (2019), BenBen Ghana: Empowering citizens by providing land security, https://www.cta.int/en/blog/all/article/benben-ghana-empowering-citizens-by-providing-landsecurity-sid0ff22d6c6-ddf1-400c-a6cb-395c42d4e468.

Builtin (2020), Blockchain Technology Defined, https://builtin.com/blockchain.

Business Insider by Pulse (2019), How Seso Global plans to unlock $\$ 100$ billion trapped in Nigeria's real estate market using blockchain technology, https://www.pulse.ng/bi/tech/howseso-global-plans-to-unlock-dollar100-billion-trapped-in-nigerias-real-estate/0z2epv5.

CARE (2017), Adaptable and effective: Cash in the face of multi-dimensional crisis Lessons from Zimbabwe, https://insights.careinternational.org.uk/media/k2/attachments/CARE CTPlessons-from-Zimbabwe-summary 2017 web.pdf. 
Christie, A. (2020), "Can Distributed Ledger Technologies Promote Trust for Charities? A Literature Review", Frontiers in Blockchain, Vol. 3, http://dx.doi.org/10.3389/fbloc.2020.00031.

Coinfy (2017), Hack the Future of Development Aid, https://www.bond.org.uk/sites/default/files/resourcedocuments/hack the future december 2017.pdf.

ConsenSys (2020), , https://consensys.net/.

ConsenSys (2020), Blockchain in Digital Identity, https://consensys.net/blockchain-usecases/digital-identity/.

ConsenSys (2020), How Does a Blockchain Work?, https://consensys.net/knowledge-base/howdoes-a-blockchain-work/.

ConsenSys (2019), Revolutionising Humanitarian cash transfers in Vanuatu, https://cdn2.hubspot.net/hubfs/4795067/Project\%20Unblocked\%20Cash\%20/ProjectUnblocked-Cash-ConsenSys.pdf.

Coppi, G. and L. Fast (2019), "Blockchain and Distributed Ledger Technology in the Humanitarian Sector", Overseas Development Institute, HPG Commissioned Report, https://www.odi.org/sites/odi.org.uk/files/resource-documents/12605.pdf.

Creti, P. and S. Jaspars (2006), Planning cash transfer programmes, Oxfam, https://books.google.ch/books?id=IRd5N7or9lcC\&pg=PA8\&lpg=PA8\&dq=cash+assistance+i mproves+choice+and+dignity\&source=bl\&ots=bEdkkqoJYN\&sig=ACfU3U1yGOAJYJOw3hB2 0Numd2SZ9tc Bg\&hl=en\&sa=X\&ved=2ahUKEwjm46P416vqAhXIBWMBHYDHBCgQ6AEwB HoECAkQAQ\#v=onepage $\& q=$ cash\%20a.

Creydt, M. and M. Fischer (2019), "Blockchain and more - Algorithm Driven Food Traceability", Food Control, Vol. 1.105, https://doi.org/10.1016/j.foodcont.2019.05.019.

Crypto Gibraltar (2020), Stellar (XLM) based Akon's Akoin to Digitize Medical Access in Rwanda, http://www.cryptogib.gi/stellar-xlm-based-akons-akoin-to-digitize-medical-access-in-rwandal.

CSO Partnership for Development Effectiveness (2020), .

Denis Le Sève, M., N. Mason and D. Nassiry (2018), "Delivering blockchain's potential for environmental sustainability", ODI Briefing Note, https://www.odi.org/publications/11206delivering-blockchain-s-potential-environmental-sustainability.

Devex (2020), Covid-19 - it's time to take cash to the next level, https://www.devex.com/news/opinion-covid-19-it-s-time-to-take-cash-to-the-next-level-97394.

Doocy, S. and H. Tappis (2017), "Cash-based approaches in humanitarian emergencies: a systematic review", Campbell Systematic Reviews, Vol. 13/1, pp. 1-200, http://dx.doi.org/10.4073/csr.2017.17.

Dwivedi, A. et al. (2019), "A Decentralized Privacy-Preserving Healthcare Blockchain for loT, SENSORS,", Vol. 19, no.2.

Energy Storage News (2020), Smart neighbourhood, smart microgrid, https://www.energystorage.news/blogs/smart-neighbourhood-smart-microgrid. 
European Blockchain Observatory and Forum (2018), Blockchain for Governance and Public Services.

Financial Action Task Force (2019), Guidance for a Risk-Based Approach to Virtual Assets and Virtual Asset Service Providers, https://www.fatfgafi.org/publications/fatfrecommendations/documents/guidance-rba-virtual-assets.html.

Finextra (2020), Blockchain and Interoperability: key to mass adoption, https://www.finextra.com/blogposting/18972/blockchain-and-interoperability-key-to-massadoption.

Forbes (2020), Coronavirus Has Made Akon's \$6 Billion Crypto-Powered Real Life Wakanda in Senegal More Necessary, https://www.forbes.com/sites/billybambrough/2020/10/05/coronavirus-has-made-akons-6billion-crypto-powered-real-life-wakanda-in-senegal-more-necessaryl.

GIZ Blockchain Lab (2019), Blockchain: A World Without Middlemen? Promise and Practice of Distributed Governance, https://www.giz.de/en/downloads/giz2019 en blockchain world without middleman.pdf.

Global Digital Finance (2019), Global Digital Council appoints advisory council and executive director, launches global membership and releases additional industry code of conduct principles to drive global industry standards in cryptoassets.

GSMA (2017), Blockchain for Development: Emerging Opportunities for Mobile, Identity and Aid, https://www.gsma.com/mobilefordevelopment/wp-content/uploads/2017/12/Blockchain-forDevelopment.pdf.

Hacibeyoglu, C. (2008), Case Study of Ghana -- When enough is enough.

Harvey, P. and S. Pavanello (2018), Multi-Purpose Cash and sectoral outcomes: a review of evidence and learning, UNHCR, https://www.unhcr.org/5b28c4157.pdf.

Hutt, R. (2016), "All you need to know about blockchain, explained simply", World Economic Forum, https://www.weforum.org/agenda/2016/06/blockchain-explained-simply/.

ICO.li (2019), How do blockchain networks validate data?.

IFC (2017), Blockchain: Opportunities for Private Enterprises in Emerging Markets.

Institute of Development Studies (2017), "Blockchain for Development - Hope or Hype?", Rapid Response Briefing 17.

International Aid Transparency Initiative (2020), International Aid Transparency Initiative.

International Federation of Red Cross and Red Crescent Societies (2018), "Learning Review: Open Loop Cash Transfer Pilot Project".

International Finance (2020), The Rise of Crypto Adoption in Africa, https://internationalfinance.com/the-rise-of-crypto-adoption-in-africal.

Investopedia (2020), Crypto Regulatory Sandbox, https://www.investopedia.com/terms/c/crypto[66] regulatory-sandbox.asp. 
Jenkinson, G. (2019), Ethereum Classic 51\% Attack, https://cointelegraph.com/news/ethereumclassic-51-attack-the-reality-of-proof-of-work.

Joudrey, S. (2019), What is a Private Blockchain?, https://hedgetrade.com/what-is-a-privateblockchain/.

Jun, M. (2018), "Blockchain Government - A Next Form of Infrastructure for the Twenty-First Century", Journal of Open Innovatin, Technology, Market and Complexity, Vol. 4, no. 1.

Juskalian, R. (2018), Inside the Jordan refugee camp that runs on blockchain, MIT Technology review, https://www.technologyreview.com/2018/04/12/143410/inside-the-jordan-refugeecamp-that-runs-on-blockchain/.

Karuhanga, J. (2018), Blockchain technology tipped to boose healthcare delivery in Africa, https://www.newtimes.co.rw/news/blockchain-technology-tipped-boost-healthcare-deliveryafrica.

Kenna, S. (2017), How Blockchain Technology Is Helping Syrian Refugees, Huffpost, https://www.huffingtonpost.com.au/2017/11/05/how-blockchain-technology-is-helping-syrianrefugees a 23267543/? guc consent skip $=1594815884$.

KfW (2019), Project Information, KfW Development Bank, https://www.kfwentwicklungsbank.de/PDF/Entwicklungsfinanzierung/ThemenNEU/Digitalisierung/2018 TruBudget.pdf.

Khan, F. (2019), What are the different types of DLTs \& how they work?, https://www.datadriveninvestor.com/2019/02/14/what-are-the-different-types-of-dlts-how-theywork/\#.

Kleffmann, P. (2019), Blockchain technology for partnerships at eye-level, https://www.dandc.eu/en/article/new-digital-platform-designed-kfw-facilitates-transparent-andsecure-implementation-oda.

Klingebiel, S., T. Mahn and M. Negre (2016), "Fragmentation: A Key Concept for Development Cooperation", in The Fragmentation of Aid, Palgrave Macmillan UK, London, http://dx.doi.org/10.1057/978-1-137-55357-7 1.

Ledger Ops (2019), Blockchains Aren't Anonymous. But They Can Be., https://ledgerops.com/blog/blockchains-arent-anonymous-but-they-can-be-05-01-2019/.

Lucsok, P. (2018), Fighting hunger with blockchain, Partity, https://www.parity.io/fighting-hungerwith-blockchain/.

Marr, B. (2020), What is The Difference Between Blockchain and Bitcoin?, https://www.bernardmarr.com/default.asp?contentID=1849\#: :text=Decentralised.,-Distributed.\&text=So\%2C\%20Bitcoin\%20was\%20the\%20first,are\%20so\%20often\%20used\% 20interchangeably.\&text=So\%20although\%20Bitcoin \%20uses $\% 20$ blockchain, is $\% 20$ more $\% 2$ 0than\%20just\%20Bitc.

Mckechnie, A. and F. Davies (2013), "Localising Aid, is it worth the risk?", Centre for Aid \& Public Expenditure. 
Medium (2017), Fintech for All: Seso founder on building a blockchain land registry for Africa, https://medium.com/@The LHoFT/fintech-for-all-seso-founder-on-building-a-blockchain-landregistry-for-africa-6909c27d141d.

Move Africa Forward (2020), Blockchain Africa Conference.

Mulligan, C. (2016), Application of Distributed Ledger Technology within Department for International Development.

Mulligan, C., P. Godsiff and A. Brunelle (2020), "Boundary Spanning in a Digital World: The Case of Blockchain", https://doi.org/10.3389/fbloc.2020.00037.

Nakamoto, S. (2009), Bitcoin: A Peer-to-Peer Electronic Cash System, https://bitcoin.org/bitcoin.pdf.

Nelson, P. (2018), A Primer on Blockchain, https://www.usaid.gov/sites/default/files/documents/15396/USAID-Primer-Blockchain.pdf.

New York Times (2020), Bitcoin Has Lost Steam. But Criminals Still Love it., https://www.nytimes.com/2020/01/28/technology/bitcoin-black-market.html.

OECD (2020), Development Co-operation Profiles, https://doi.org/10.1787/2dcf1367-en.

OECD (2020), The impact of the coronavirus (COVID-19) crisis on development finance, http://www.oecd.org/coronavirus/policy-responses/the-impact-of-the-coronavirus-covid-19crisis-on-development-finance-9de00b3b/.

OECD (2019), Managing for Sustainable Develoipment Results.

OECD (2018), OECD Blockchain Primer, https://www.oecd.org/finance/OECD-BlockchainPrimer.pdf.

OECD (2018), OECD Due Diliegnce Guidance for Responsible Business Conduct, https://mneguidelines.oecd.org/MNEguidelines RBCmatters.pdf.

OECD (2011), Busan Partnership for Effective Development Co-operation, https://www.oecd.org/development/effectiveness/busanpartnership.htm\#: :text=The\%20Busa n\%20Partnership\%20agreement\%20sets, in\%20support\%20of\%20international\%20developm ent.\&text=The\%20Global\%20Partnership\%20will\%20help,commitments\%20at\%20the\%20pol itical\%20le.

OECD (2010), Country Systems, and Why We Need to Use Them, OECD Publishing, Paris, https://dx.doi.org/10.1787/dcr-2010-en.

Office of the United Nations High Commissioner for Refugees (2020), Cash-based interventions, https://www.unhcr.org/cash-basedinterventions.htm|\#: : :text=UNHCR\%20uses\%20cash\%2Dbased\%20interventions, and\%20to \%20facilitate\%20voluntary\%20repatriation.

Oxfam (2020), , https://www.oxfam.org/en.

Parulwa, S. (2017), Blockchains, Distributed Ledgers and Funds Transfer: An Overview. 
Pisa, M. (2018), "Reassessing Expectations for Blockchain and Development", Center for Global Development Note, https://www.cgdev.org/sites/default/files/reassessing-expectationsblockchain-and-development-cost-complexity.pdf.

Popov, A. (2020), "How Industrial Sectors are Using Blockchain Technology", Forbes Technology Council, https://www.forbes.com/sites/forbestechcouncil/2020/03/12/howindustrial-sectors-are-using-blockchain-technologyl.

Power Technology (2017), The Brooklyn Micro-Grid: Blockchain Enabled Community Power, https://www.power-technology.com/features/featurethe-brooklyn-microgrid-blockchainenabled-community-power-5783564/.

Rayner, T. (2018), "How Finland is Using the Blockchain to Revolutionise FInancial Services for Refugees", Reset, Digital for Good, https://en.reset.org/blog/how-finland-using-blockchainrevolutionise-financial-services-refugees-05102018.

Reinsberg, B. (2020), "Fully automated liberalism? Blockchain technology and international cooperation in an anarchic world", http://dx.doi.org/10.1017/S1752971920000305.

Reinsberg, B. (2019), "Blockchain technology and the governance of foreign aid", Journal of Institutional Economics, http://dx.doi.org/10.1017/S1744137418000462.

Rosic, A. (2017), "What Is Hashing? [Step-by-Step Guide-Under Hood of Blockchain]", Online Hash Crack, https://blockgeeks.com/guides/what-is-hashing/.

Rosic, A. (2016), What is Blockchain Technology? A Step-by-Step Guide For Beginners, https://blockgeeks.com/guides/what-is-blockchain-technology/.

Rühmann, F. (2020), Can blockchain technology reduce the cost of remittances?, https://doi.org/10.1787/d4d6ac8f-en.

Rust, B. (2019), Unblocked Cash: piloting accelerated cash transfer delivery in Vanuatu, Oxfam, https://oxfamilibrary.openrepository.com/bitstream/handle/10546/620926/rr-unblocked-cashdelivery-vanuatu-311019en.pdf;isessionid=A25544E82B1C48EFC082C5CE7F5D961E? sequence=1.

Salmon, J. and G. Myers (2019), Blockchain and Associated Legal Issues in Emerging Markets, https://www.ifc.org/wps/wcm/connect/da7da0dd-2068-4728-b846-7cffcd1fd24a/EMCompassNote-63-Blockchain-and-Legal-Issues-in-EmergingMarkets.pdf?MOD=AJPERES\&CVID=mxocw9F.

Sayers, S. (2017), Code to Integrity, Digital avenues to anti-corruption - also for her!, http://file:///C:/Users/Stout E/Downloads/Code\%20to\%20Integrity Enkeltsider Web\%20(1).p df.

Sempo (2020), , https://withsempo.com/.

Seso Global (2020), Home.

Slavin, T. (2019), How AB InBev is using blockchain to improve the lives of smallholder farmers, https://www.reutersevents.com/sustainability/how-ab-inbev-using-blockchain-improve-livessmallholder-farmers. 
Smith, A. (2019), How the World Food Programme uses blockchain to better serve refugees, ITU News, https://news.itu.int/how-the-world-food-programme-uses-blockchain-to-better-serverefugees/.

solshare (2020), Who We Are.

Tapscott, D. and A. Tapscott (2016), Blockchain Revolution: How the Technology Behind Bitcoin is Changing Money, Business and the World.

Toolbox (2020), 3 Reasons Bitcoin is an Attractive Hedge for Inflation, https://www.toolbox.com/finance/fintech/guest-article/3-reasons-bitcoin-is-an-attractivehedge-forinflation/\#: :text=Bitcoin\%20is\%20anti\%2Dinflation.,on\%20the\%20value\%20of\%20decentrali zation.

United Nations (2019), Remittances matter: 8 facts you don't know about the money migrants send back home, https://www.un.org/development/desa/en/news/population/remittancesmatter.html.

United Nations (2015), Addis Ababa Action Agenda, https://www.un.org/esa/ffd/wpcontent/uploads/2015/08/AAAA Outcome.pdf.

United Nations (2015), Sustainable Development Goals, https://www.who.int/sdg/en/\#: : text=Tracking\%20progress\%20towards\%20Universal\%20Hea th\%20Coverage\&text=The\%20United\%20Nations\%20Sustainable\%20Development, achieve \%20by\%20the\%20year\%202030.\&text=Almost\%20all\%20of\%20the\%20other, will\%20contrib ute $\% 20$ to $\% 20$ healt.

United Nations (1948), Universal Declaration on Human Rights, https://www.un.org/en/udhrbook/pdf/udhr booklet en web.pdf.

United Nations Capital Development Fund (2020), Financial Inclusion and the SDGs, https://www.uncdf.org/financial-inclusion-and-the-sdgs.

United Nations Economic and Social Commission for Asia and the Pacific (2017), Digital and Virtual Currencies for Sustainable Development, https://www.unescap.org/sites/default/files/ESCAP DV Currency 0.pdf.

United Nations Framework Convention on Climate Change (2016), Paris Agreement.

United Nations Global Compact (2004), Ten Principles, https://www.unglobalcompact.org/whatis-gc/mission/principles.

United Nations Office on Drugs and Crime (2017), In Just Two Decades, Technology Has Become a Cornerstone of Criminality.

Vallee, B. and Y. Yu (2020), "Seso Global: Building a Blockchain-enabled Property Market in Nigeria", Harvard Business School N9220-055.

World Bank (2020), COVID-19: Remittance Flows to Shrink 14\% by 2021, https://www.worldbank.org/en/news/press-release/2020/10/29/covid-19-remittance-flows-toshrink-14-by-2021. 
World Bank (2020), Small and Medium Enterprises' Finance, https://www.worldbank.org/en/topic/smefinance.

World Bank (2018), The global identification challenge: Who are the 1 billion people without proof of identity?, https://blogs.worldbank.org/voices/global-identification-challenge-who-are1-billion-people-without-proof-identity.

World Bank (2016), Investing Back Home: The Potential Economic Role of the Caribbean Diaspora.

World Economic Forum (2020), 3 Ways Blockchain Can Accelerate Sustainable Development, https://www.weforum.org/agenda/2020/09/3-ways-blockchain-can-contribute-to-sustainabledevelopment/.

World Economic Forum (2020), How the Massive Plan to Deliver the Covid-19 Vaccine could make History - and Leverage Blockchain Like Never Before, https://www.weforum.org/agenda/2020/07/blockchain-role-in-distributing-covid-19-vaccinecould-make-historyl.

World Economic Forum (2020), Presidio Principles, Foundational Values for a Decentralised Future, http://www3.weforum.org/docs/WEF Presidio Principles 2020.pdf.

World Food Programme (2020), Building Blocks: Blockchain for zero hunger, https://innovation.wfp.org/project/buildingblocks\#: :text=Built\%20on\%20a\%20private\%2C\%20permissioned,and\%20privacy\%20for\%2 0Syrian\%20refugees.

World Food Programme (2017), Annual report 2017 United Nations World Food Programme Innovation accelerator, https://innovation.wfp.org/year-review-2017/docs/WFP-innovationaccelerator-2017-annual-report.pdf.

Zambrano, R., A. Young and S. Verhulst (2018), Connecting refugees to aid through blockchainenabled ID management: World Food Programme's Building Blocks, GovLab, https://blockchan.ge/blockchange-resource-provision.pdf. 